\title{
Periodic Responses of a Rotating Hub-Beam System with a Tip Mass under Gravity Loads by the Incremental Harmonic Balance Method
}

\author{
D. W. Zhang, ${ }^{1}$ J. K. Liu, ${ }^{1}$ J. L. Huang ${ }^{(D},{ }^{1}$ and W. D. Zhu ${ }^{2}$ \\ ${ }^{1}$ Department of Applied Mechanics and Engineering, Sun Yat-sen University, Guangzhou 510275, China \\ ${ }^{2}$ Department of Mechanical Engineering, University of Maryland, Baltimore County, 1000 Hilltop Circle, Baltimore, MD 21250, USA
}

Correspondence should be addressed to J. L. Huang; huangjl@mail.sysu.edu.cn

Received 8 August 2017; Revised 22 November 2017; Accepted 9 January 2018; Published 13 March 2018

Academic Editor: Francesco Pellicano

Copyright (c) 2018 D. W. Zhang et al. This is an open access article distributed under the Creative Commons Attribution License, which permits unrestricted use, distribution, and reproduction in any medium, provided the original work is properly cited.

\begin{abstract}
Dynamic characteristics of a flexible hub-beam system with a tip mass under gravity loads are investigated. The slope angle of the centroid line of the beam is utilized to describe its motion. Hamilton's principle is used to derive the equations of motion and their boundary conditions. By using Lagrange's equations, spatially discretized equations based on assumed mode method are derived, and the equations of motion are expressed in nondimensional matrix form. The incremental harmonic balance (IHB) method is used to solve for periodic responses of a high-dimensional model of the rotating hub-beam system with a tip mass for which convergence is reached. A frequency equation is derived giving the relationship between the nondimensional natural frequencies and three nondimensional parameters, that is, the rotating angular velocity, the tip mass, and the hub radius ratio. A comparative study is performed for nonlinear frequency responses of the system with a tip mass under different values of tip masses and damping ratios.
\end{abstract}

\section{Introduction}

A number of systems in the fields of navigation and mechanical engineering can be modeled as rotating hub-beam system with a tip mass. For example, flexible manipulators, spacecraft structures, and cranes carrying moving loads can be studied in this way. In these systems, the beams carrying a tip mass are rotating in a horizontal plane with the whole systems mounted on a rotating hub. In order to study their dynamic characteristics, the rotating beam systems are simplified as a rotating hub-beam system with a tip mass model. In recent years much attention has been placed on linear and nonlinear dynamic characteristics of rotating beam with a tip mass, and earlier work has been done with the linear analysis. For instance, Conrad and Morgül [1] used linearized feedback law to study the stabilization of a flexible beam with a tip mass. Rao [2] derived the time-dependent equations of motion that governs the vibration of an Euler-Bernoulli beam; it was used to obtain the linear dynamic response of the beam under moving load mass. Demetriou [3] presented a method for construction of observer for linear second-order lumped and distributed parameter systems using parameterdependent Lyapunov functions. Furta [4] proved that the attached point mass on a thin elastic beam plays a destabilizing role for any values of the problem parameters and studied the dynamical stability of the rectilinear shape of the beam by means of the direct Lyapunov method. In [5], the extended Hamilton principle was employed to derive the equations of motion of a rotating beam with a tip mass undergoing coupled torsional-bending vibrations and analyzing the exact frequencies leading to a better control of the system.

Recently, the nonlinear vibration of a rotating beam with a tip mass has been studied by numerous researchers. Yang et al. [6] presented a finite element model for a flexible hubbeam system with a tip mass where viscous damping of the hub and the air drag force were considered. They showed that the traditional linear model cannot account for the dynamic stiffening and it may lead to erroneous results in high-speed systems. Simulation results on the vibration measurements 
of the rotating flexible beam carrying a tip mass for different combinations of shaft and root flexibilities and arm properties were reported by Ismail et al. [7]. Gregory et al. [8] have pointed out the shortcomings of the sequential single-axis vibration method. They have also reported evidence of differences in failure modes and fatigue life for multiaxial loadings versus single-axis inputs by utilizing multiaxial electrodynamic shakers. Sunar and Al-Bedoor [9] tested the suitability of a piezoelectric (PZT) sensor in measuring vibrations of the rotating beams. A comparison between the results of finite element and experimental was reported and indicated that the root-embedded PZT sensor can be effectively used in measurements of blade vibration. In [10], Sinha et al. investigated a simultaneously precessing and nutating beam with a tip mass using a variant of Hill's method and found that the stability of an only precessing beam depends on the inclination of the beam-centerline with the axis of precession. Patil and Gandhi [11] demonstrated chaotic behavior for an inverted flexible pendulum with a tip mass on a cart system; the dynamic model was validated with experimental data for a couple of cases of beam excitation. Li et al. [12] established the dynamic equations of the rigid-flexible coupling system in a noninertial coordinate system; the numerical simulation results showed that dynamic stiffening is produced by the coupling effect of the centrifugal inertial load distributed on the flexible beam and the transverse vibration deformation of the beam. Yong et al. [13] studied the rigid-flexible coupling system with a hub and concentrated mass; they utilized the second Lagrange equation and the assumed mode method to establish the dynamic equations of the system. The results showed that the concentrated mass mainly suppresses the bending vibration of the beam and exhibits damping characteristics.

In a rotating hub-beam system with a tip mass, the effect of the gravity load of beam was not accounted for in the previous literature [14]. As the size and height of rotating machineries such as manipulators and cranes increase, it is necessary to consider the effect of the gravity load of the beam during the rotation. Park and Kim [15] derived the equations of motion of the rotating beam which include all dynamic effects and mainly studied the effects of curvature and tip mass that can change the dynamic response of the beam. Flatness based controller design techniques for a rotating hub-beam system with a payload attached to the tip of the beam in a gravitational field were utilized in [16]. Cai et al. [17] made a comparison between the first-order approximation coupling model and the zerothorder approximation coupling model when prescribed torque drives the beam with a tip mass, which takes into account the influence of the gravitational force filed of the beam in the analysis of the dynamic characteristics of the system. As for a flexible hub geometrical nonlinearity beam with a tip mass, Emam [18] employed a flexural model to study the dynamic responses of a flexible hub geometrical beam with a tip mass of which the hub is restrained by a translational and a rotational spring; such a model accounts for the geometrical coupling between the axial and lateral deformations. If gravity of the beam is much less than the gravity of tip mass, it is not necessary to consider the effect of the gravity load of beam [19].

The main objective of this work is to use the IHB method to analyze nonlinear dynamic responses of a rotating hubbeam system with a tip mass for which convergence is reached. The vibration analysis is performed under the slope angle model with five included trial functions, and the model considers the effects of gravity loads of the beam and tip mass during the rotation. To deal with the rotating hub-beam system with a tip mass, the IHB method is applied, which was first introduced by Lau and Cheung [20, 21]. The IHB method was successfully applied to the analysis of nonlinear structural vibrations problems. Xu and Zhu [22] used the IHB method to determine parametric instability boundaries of a parametrically excited system. In order to improve calculation efficiency of the IHB method, the fast Fourier transform (FFT) was used to efficiently calculate coefficients of a discrete Fourier transform in practice [23]. Wang and Zhu [24] developed a modified IHB method that incorporated FFT and Broyden's method to obtain the dynamic responses of beltdrive system with a noncircular sprocket. The configuration of the rotating beam is described by the slope angle of its centroid line; the slope angle model has been widely used in studying the nonlinear dynamics [25]. The nonlinear dynamic equation of the rotating hub-beam system with a tip mass is derived in Section 2 using Hamilton's principle. Spatially discretized equations of the slope angle model neglecting the fourth- and higher-order nonlinear terms are derived by Lagrange's equations in Section 3. The IHB method for calculating the periodic responses of the rotating hub-beam system with a tip mass is formulated in Section 4 . The critical buckling load of the rotating beam under an axial tip mass is calculated in Section 5. Natural frequencies of the rotating beam with a constant rotating angular velocity are calculated in Section 6.1 using the slope angle model, and compared with those from the dynamical model in [14]. Nonlinear frequency responses of the system with a tip mass for different numbers of trial functions are calculated in Section 6.2. The influences of tip mass and damping ratio on nonlinear frequency responses are studied in Sections 6.3 and 6.4, respectively.

\section{Equations of Motion}

A schematic diagram of a rotating beam with a tip mass is shown in Figure 1, a rotating planar beam is attached to a rigid hub of radius $r_{0}$, and the beam rotates in a vertical plane around the central axis $O$ in the inertial coordinates $X Y$. The effects of gravity loads of the beam and tip mass are considered. The beam has a slender shape that the shear deformation effect and rotary inertia of the beam are ignored. The position of a cross section of the beam is described by the arc-length coordinate $s$ along its centroid line. For the whole beam, the bending stiffness $E I(s)$, cross-section area $A(s)$, and linear mass density $\rho(s)$ can be approximated as constant. $L$ is the length of the beam, and $m_{t}$ is the tip mass at the end of the beam. The hub rotates with a given rotating angular velocity $\Omega(t)$, where $t$ is time; the gravitational acceleration is 


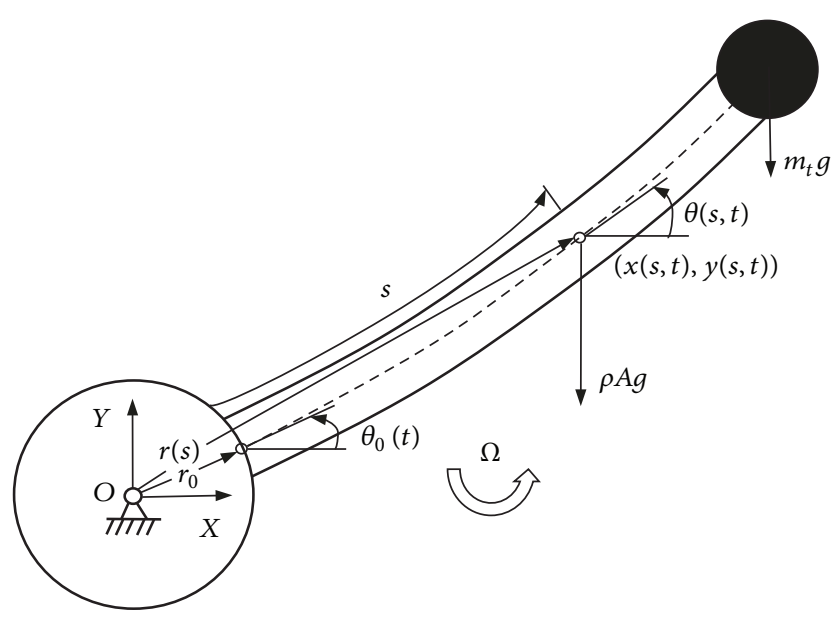

FIGURE 1: Configuration of a rotating hub-beam system with a tip mass.

$g=9.81 \mathrm{~m} / \mathrm{s}^{2} \cdot \theta_{0}(t)$ represents the space attitude motion of hub, and

$$
\theta_{0}(t)=\int_{0}^{t} \Omega(\chi) d \chi
$$

The slope angle of the beam at the position $s$ is expressed as $\theta(s, t)$, then $\theta(0, t)=\theta_{0}(t)$.

The Cartesian coordinates of any one particle of the beam with the arc-length coordinate $s$ are

$$
\begin{aligned}
& x(s, t)=r_{0} \cos \theta_{0}(t)+\int_{0}^{s} \cos \theta(\varsigma, t) d \varsigma, \\
& y(s, t)=r_{0} \sin \theta_{0}(t)+\int_{0}^{s} \sin \theta(\varsigma, t) d \varsigma .
\end{aligned}
$$

The Cartesian coordinates $(x, y)$ are functions of $\theta(s, t)$; hence, one can use $\theta(s, t)$ to describe the configuration of the beam. The kinetic energy of the rotating hub-beam system with a tip mass is

$$
\begin{aligned}
K= & \frac{1}{2} \int_{0}^{L} \rho(s) A(s)\left[\dot{x}^{2}(s, t)+\dot{y}^{2}(s, t)\right] d s \\
& +\frac{1}{2} m_{t}\left[\dot{x}^{2}(L, t)+\dot{y}^{2}(L, t)\right]+\frac{1}{2} J_{h} \dot{\theta}_{0}^{2},
\end{aligned}
$$

where a dot over $x$ and $y$ denotes differentiation of them with respect to $t$ and $J_{h}$ is the rotary inertia of the hub.

The potential energy of the system with a tip mass can be expressed as

$$
\begin{aligned}
V= & U+V_{g} \\
= & \frac{1}{2} \int_{0}^{L} E I(s)\left[\theta_{s}(s, t)\right]^{2} d s+\int_{0}^{L} \rho(s) A(s) g y(s) d s \\
& +m_{t} g y(L),
\end{aligned}
$$

where $U$ and $V_{g}$ represent the elastic potential energy and the gravitational potential energy, respectively. For brevity in expression, the variables $\theta_{0}(t)$ and $\phi(s, t)$ are written as $\theta_{0}$ and $\phi$. Substituting (2) into (3) and (4), one obtains the variations of the kinetic energy and the potential energy

$$
\begin{aligned}
\delta K & =\int_{0}^{L}\left\{\left[\int_{s}^{L} \rho(\varsigma) A(\varsigma) \dot{y}(\varsigma, t) d \varsigma \cos \theta(s, t)\right.\right. \\
& \left.-\int_{s}^{L} \rho(\varsigma) A(\varsigma) \dot{x}(\varsigma, t) d \varsigma \sin \theta(s, t)\right] \delta \dot{\theta} \\
& -\left[\int_{s}^{L} \rho(\varsigma) A(\varsigma) \dot{x}(\varsigma, t) d \varsigma \cos \theta(s, t)\right. \\
& \left.\left.+\int_{s}^{L} \rho(\varsigma) A(\varsigma) \dot{y}(\varsigma, t) d \varsigma \sin \theta(s, t)\right] \dot{\theta} \delta \theta\right\} d s \\
& +\int_{0}^{L}\left\{m_{t}[\dot{y}(L, t) \cos \theta(s, t)-\dot{x}(L, t) \sin \theta(s, t)] \delta \dot{\theta}\right. \\
& -m_{t}[\dot{x}(L, t) \cos \theta(s, t)+\dot{y}(L, t) \sin \theta(s, t)] \\
& \cdot \dot{\theta} \delta \theta\} d s, \\
\delta V & =\int_{0}^{L} E I(s) \theta_{s}(s, t) \delta \theta_{s}(s, t) d s+\int_{0}^{L} \rho(s) A(s) \\
& \cdot g \delta y(s) d s+m_{t} g \delta y(L)=\left.E I(s) \theta_{s}(s, t) \delta \theta(s, t)\right|_{0} ^{L} \\
& -\int_{0}^{L}\left[E I(s) \theta_{s}(s, t)\right]_{s} \delta \theta(s, t) d s+\int_{0}^{L} g \int_{s}^{L} \rho(\varsigma) \\
& +A(\varsigma) d \varsigma \cos \theta(s, t) \delta \theta(s, t) d s \\
& +m_{t} g \int_{0}^{L} \cos \theta(s, t) \delta \theta(s, t) d s,
\end{aligned}
$$

where $\left[E I(s) \theta_{s}(s, t)\right]_{s}$ denotes partial differentiation of $E I(s) \theta_{s}(s, t)$ with respect to $s$. The boundary term $E I(s) \theta_{s}(s$, t) $\left.\delta \theta(s, t)\right|_{0} ^{L}=0$, which gives the following boundary conditions:

$$
\begin{aligned}
\theta(0, t) & =\theta_{0}(t), \\
\theta_{s}(L, t) & =0 .
\end{aligned}
$$

The relationship between $\theta(s, t)$ and $\theta_{0}(t)$ can be expressed as follows:

$$
\theta(s, t)=\theta_{0}(t)+\phi(s, t)
$$

where $\phi$ is the slope angle of the centroid line of the beam measured from a rotating axis attached to the hub. The boundary conditions in (6) can then become

$$
\begin{gathered}
\phi(0, t)=0, \\
\phi_{s}(L, t)=0,
\end{gathered}
$$

where $\theta_{s}(s, t)$ and $\phi_{s}(s, t)$ denote partial differentiation of $\theta(s$, $t)$ and $\phi(s, t)$ with respect to $s$, respectively. Using Hamilton's 
principle and (7), the governing integro-partial differential equation is obtained as follows:

$$
\begin{aligned}
& \int_{0}^{L} \rho(s) A(s)\left\{\int_{0}^{s} \sin \left[\theta_{0}(t)+\phi(\varsigma, t)\right][\dot{\Omega}(t)+\ddot{\phi}(\varsigma, t)] d \varsigma\right. \\
& \quad \cdot \int_{0}^{s} \sin \left[\theta_{0}(t)+\phi(\varsigma, t)\right] d \varsigma \\
& +\int_{0}^{s} \cos \left[\theta_{0}(t)+\phi(\varsigma, t)\right][\dot{\Omega}(t)+\ddot{\phi}(\varsigma, t)] d \varsigma \\
& \quad \int_{0}^{s} \cos \left[\theta_{0}(t)+\phi(\varsigma, t)\right] d \varsigma \\
& \quad-\int_{0}^{s}\left[\ddot{x}_{0} \sin \left[\theta_{0}(t)+\phi(\varsigma, t)\right]-\ddot{y}_{0} \cos \left[\theta_{0}(t)+\phi(\varsigma, t)\right]\right] d \varsigma \\
& \left.+\int_{0}^{s} g \cos \phi(\varsigma, t) d \varsigma\right\} d s+\left[E I(s) \phi_{s}(s, t)\right]_{s} \\
& +m_{t}\left\{\int_{0}^{L} \sin \left[\theta_{0}(t)+\phi(s, t)\right]\left[\dot{\Omega}^{\prime}(t)+\ddot{\phi}(s, t)\right] d s\right. \\
& \quad \cdot \int_{0}^{L} \sin \left[\theta_{0}(t)+\phi(s, t)\right] d s+\int_{0}^{L} \cos \left[\theta_{0}(t)+\phi(s, t)\right] \\
& +\left[\dot{\Omega}_{0}^{L}(t)+\ddot{\phi}(s, t)\right] d s \int_{0}^{L} \cos \left[\theta_{0}(t)+\phi(s, t)\right] d s \\
& +\int_{0}^{L}\left[\ddot{x}_{0} \sin \left[\theta_{0}(t)+\phi(s, t)\right]-\ddot{y}_{0} \cos \left[\theta_{0}(t)+\phi(s, t)\right]\right] d s \\
& \left.+\int_{0}^{L} \cos \phi(s, t) d s\right\}=0 .
\end{aligned}
$$

Equation (9) presents a new rigid-flexible dynamic model of a rotating hub-beam system with a tip mass described by rotating angle of the hub $\theta_{0}(t)$ and slope angle $\phi(s, t)$.

\section{Spatial Discretization}

Assume the solution of (9) can be expressed by

$$
\phi(s, t)=\sum_{j=1}^{n} \psi_{j}(s) q_{j}(t)=\psi^{T}(s) \mathbf{q}(t)
$$

where the superscript $T$ denotes transpose of a vector or matrix, $\psi_{j}(s)=(1 / \sqrt{2}) \sin [(j-1 / 2) \pi(s / L)]$ are trial functions used, which are eigenvalue functions of a fixed-free rod, $n$ is the number of included trial functions, $q_{j}(t)$ are the generalized coordinates, $\psi=\left[\begin{array}{llll}\psi_{1} & \psi_{2} & \cdots & \psi_{n}\end{array}\right]^{T}$, and $\mathbf{q}=$ $\left[\begin{array}{llll}q_{1} & q_{2} & \cdots & q_{n}\end{array}\right]^{T}$.

The position vector of the particle and tip mass in the inertial coordinate system $O X Y$ can be expressed by $\mathbf{r}_{p}, \mathbf{r}_{t}$, and $\mathbf{r}_{0}$, and their Cartesian coordinates corresponding to arclength coordinate $s$ are as follows, respectively:

$$
\begin{aligned}
& \mathbf{r}_{p}(s, t)=\left[\begin{array}{ll}
x(s, t) & y(s, t)
\end{array}\right]^{T}=\mathbf{r}_{0}(t)+\int_{0}^{s} \mathbf{t}(\varsigma, t) d \varsigma \\
& \mathbf{r}_{t}(L, t)=\left[\begin{array}{ll}
x(L, t) & y(L, t)
\end{array}\right]^{T}=\mathbf{r}_{0}(t)+\int_{0}^{L} \mathbf{t}(\varsigma, t) d \varsigma,
\end{aligned}
$$

$$
\begin{aligned}
\mathbf{r}_{0}(t) & =\left[\begin{array}{ll}
x_{0}(t) & y_{0}(t)
\end{array}\right]^{T} \\
& =\left[\begin{array}{ll}
r_{0} \cos \theta_{0}(t) & r_{0} \sin \theta_{0}(t)
\end{array}\right]^{T},
\end{aligned}
$$

where $\mathbf{t}(s, t)=[\cos \theta(s, t) \sin \theta(s, t)]^{T}$ is a vector tangent to the centroid line of the beam at the base. By differentiating (11) and (12) with respect to $t$, one obtains the velocity of the particle and tip mass

$$
\begin{aligned}
& \dot{\mathbf{r}}_{p}(s, t)=\dot{\mathbf{r}}_{0}(t)+\Omega(t) \mathbf{d}_{p}(s, t)+\boldsymbol{\Theta}_{p}(s, t) \dot{\mathbf{q}}(t), \\
& \dot{\mathbf{r}}_{t}(L, t)=\dot{\mathbf{r}}_{0}(t)+\Omega(t) \mathbf{d}_{t}(L, t)+\boldsymbol{\Theta}_{t}(L, t) \dot{\mathbf{q}}(t),
\end{aligned}
$$

where

$$
\begin{aligned}
& \mathbf{d}_{p}(s, t)=\int_{0}^{s} \mathbf{n}(\varsigma, t) d \varsigma, \\
& \mathbf{d}_{t}(L, t)=\int_{0}^{L} \mathbf{n}(\varsigma, t) d \varsigma, \\
& \Theta_{p}(s, t)=\int_{0}^{s} \mathbf{n}(\varsigma, t) \psi^{T}(\varsigma) d \varsigma, \\
& \Theta_{t}(L, t)=\int_{0}^{L} \mathbf{n}(\varsigma, t) \psi^{T}(\varsigma) d \varsigma
\end{aligned}
$$

in which

$$
\mathbf{n}(s, t)=\left[\begin{array}{lll}
-\sin \theta(s, t) & \cos \theta(s, t)
\end{array}\right]^{T} .
$$

The trigonometric functions $\sin \theta(s, t)$ and $\cos \theta(s, t)$ in (11) and (12) are approximated using Taylor expansion around $\theta_{0}$; one obtains

$$
\begin{aligned}
\sin \theta(s, t) \approx & S(t)+C(t) \psi^{T}(s) \mathbf{q}(t) \\
& -\frac{1}{2} S(t) \mathbf{q}^{T}(t) \psi(s) \psi^{T}(s) \mathbf{q}(t), \\
\cos \theta(s, t) \approx & C(t)-S(t) \psi^{T}(s) \mathbf{q}(t) \\
& -\frac{1}{2} C(t) \mathbf{q}^{T}(t) \psi(s) \psi^{T}(s) \mathbf{q}(t),
\end{aligned}
$$

where

$$
\begin{aligned}
& C(t)=\cos \theta_{0}(t), \\
& \mathrm{S}(t)=\sin \theta_{0}(t) .
\end{aligned}
$$

The tip mass was considered to be a particle so that the moment of inertia of tip mass is set to be zero. The kinetic energy of the beam and tip mass can be approximated by neglecting the nonlinear terms over fourth order

$$
\begin{aligned}
T= & \frac{1}{2} \int_{0}^{L} \rho(s) A(s) \dot{\mathbf{r}}_{p}^{T}(s, t) \dot{\mathbf{r}}_{p}(s, t) d s+\frac{1}{2} m_{t} \dot{\mathbf{r}}_{t}^{T}(L, t) \\
& \cdot \dot{\mathbf{r}}_{t}(L, t)=\frac{1}{2} \int_{0}^{L} \rho(s) A(s)\left[\dot{\mathbf{r}}_{0}^{T}(t) \dot{\mathbf{r}}_{0}(t)\right. \\
& +2 \Omega(t) \mathbf{d}_{p}^{T}(s, t) \dot{\mathbf{r}}_{0}(t)+2 \dot{\mathbf{r}}_{0}^{T}(t) \boldsymbol{\Theta}_{p}(s, t) \dot{\mathbf{q}}(t) \\
& +\Omega^{2}(t) \mathbf{d}_{p}^{T}(s, t) \mathbf{d}_{p}(s, t) \\
& +2 \Omega(t) \mathbf{d}_{p}^{T}(s, t) \boldsymbol{\Theta}_{p}(s, t) \dot{\mathbf{q}}(t)
\end{aligned}
$$




$$
\begin{aligned}
& \left.+\dot{\mathbf{q}}^{T}(t) \Theta_{p}^{T}(s, t) \Theta_{p}(s, t) \dot{\mathbf{q}}(t)\right] d s+\frac{1}{2} \\
& \cdot m_{t}\left[\dot{\mathbf{r}}_{0}^{T}(t) \dot{\mathbf{r}}_{0}(t)+2 \Omega(t) \mathbf{d}_{t}^{T}(L, t) \dot{\mathbf{r}}_{0}(t)+2 \dot{\mathbf{r}}_{0}^{T}(t)\right. \\
& \cdot \boldsymbol{\Theta}_{t}(L, t) \dot{\mathbf{q}}(t)+\Omega^{2}(t) \mathbf{d}_{t}^{T}(L, t) \mathbf{d}_{t}(L, t)+2 \Omega(t) \\
& \cdot \mathbf{d}_{t}^{T}(L, t) \boldsymbol{\Theta}_{t}(L, t) \dot{\mathbf{q}}(t)+\dot{\mathbf{q}}^{T}(t) \boldsymbol{\Theta}_{t}^{T}(L, t) \boldsymbol{\Theta}_{t}(L, t) \\
& \cdot \dot{\mathbf{q}}(t)] .
\end{aligned}
$$

The elastic potential energy of the beam can be written as

$$
\begin{aligned}
U & =\frac{1}{2} \int_{0}^{L} E I(s) \theta_{s}^{2}(s, t) d s=\frac{1}{2} \int_{0}^{L} E I(s) \phi_{s}^{2}(s, t) d s \\
& =\frac{1}{2} \mathbf{q}^{T}(t) \int_{0}^{L} E I(s) \psi_{s}(s) \psi_{s}^{T}(s) d s \mathbf{q}(t) .
\end{aligned}
$$

Gravity loads on the beam and tip mass are

$$
\begin{aligned}
& \mathbf{F}_{p g}=[0-\rho(s) g A]^{T}, \\
& \mathbf{F}_{t g}=\left[0-m_{t} g\right]^{T} .
\end{aligned}
$$

The virtual displacements of the particle on the beam and tip mass are

$$
\begin{aligned}
& \delta \mathbf{r}_{p}(s, t)=\boldsymbol{\Theta}_{p}(s, t) \delta \mathbf{q}(t), \\
& \delta \mathbf{r}_{t}(L, t)=\boldsymbol{\Theta}_{t}(L, t) \delta \mathbf{q}(t) .
\end{aligned}
$$

The virtual work done by $\mathbf{F}_{p g}$ on the beam and the virtual work done by $\mathbf{F}_{t g}$ on the tip mass are

$$
\begin{aligned}
\delta w_{p} & =\int_{0}^{L} \mathbf{F}_{p g}^{T} \delta \mathbf{r}_{p}(s, t) d s=\int_{0}^{L} \mathbf{F}_{p g}^{T} \boldsymbol{\Theta}_{p}(s, t) d s \delta \mathbf{q}(t) \\
\delta w_{t} & =\mathbf{F}_{t g}^{T} \delta \mathbf{r}_{t}(L, t)=\mathbf{F}_{t g}^{T} \boldsymbol{\Theta}_{t}(L, t) \delta \mathbf{q}(t)
\end{aligned}
$$

and generalized forces associated with gravity loads on the beam and tip mass are

$$
\begin{aligned}
& \mathbf{Q}_{p}(t)=\int_{0}^{L} \boldsymbol{\Theta}_{p}^{T}(s, t) \mathbf{F}_{p g}(s) d s, \\
& \mathbf{Q}_{t}(t)=\boldsymbol{\Theta}_{t}^{T}(L, t) \mathbf{F}_{t g} .
\end{aligned}
$$

As a matter of convenience, for a rotating beam with a constant rotating angular velocity, introduce the following dimensionless variables:

$$
\begin{aligned}
& \bar{s}=\frac{s}{L}, \\
& \bar{\omega}=\sqrt{\frac{E I}{\rho A L^{4}}},
\end{aligned}
$$

$$
\begin{aligned}
\bar{\Omega} & =\frac{\Omega}{\bar{\omega}}, \\
\bar{r}_{0} & =\frac{r_{0}}{L}, \\
\bar{g} & =\frac{\rho A g L^{3}}{E I}, \\
\bar{t} & =\bar{\omega} t \\
\bar{m}_{t} & =\frac{m_{t}}{\rho A L} .
\end{aligned}
$$

Substitute (19), (20), (24), and (25) into Lagrange's equations

$$
\frac{d}{d t}\left(\frac{\partial T}{\partial \dot{\mathbf{q}}}\right)^{T}-\left(\frac{\partial T}{\partial \mathbf{q}}\right)^{T}+\left(\frac{\partial U}{\partial \mathbf{q}}\right)^{T}=\mathbf{Q}(t)
$$

and dropping the over bar of each variable for brevity, one can obtain the following dimensionless equations of motion:

$$
\begin{aligned}
& \sum_{j=1}^{n}\left(M_{i j}+\sum_{k=1}^{n} \sum_{l=1}^{n} M_{i j k l} q_{k} q_{l}\right) \ddot{q}_{j} \\
& +\sum_{j=1}^{n}\left(C_{i j}+\Omega \sum_{k=1}^{n} C_{i j k} q_{k}+\sum_{k=1}^{n} \sum_{l=1}^{n} C_{i j k l} q_{k} \dot{q}_{l}\right) \dot{q}_{j} \\
& +\sum_{j=1}^{n}\left(K_{i j}^{11}+g K_{i j}^{12} \sin \Omega t+\Omega^{2} K_{i j}^{13}\right) q_{j} \\
& \quad+g \cos \Omega t \sum_{k=1}^{n} K_{i j k} q_{k} q_{j}+\Omega^{2} \sum_{j=1}^{n} \sum_{k=1}^{n} \sum_{l=1}^{n} K_{i j k l} q_{k} q_{l} q_{j} \\
& =g f_{i} \cos \Omega t,
\end{aligned}
$$

where $M_{i j}, K_{i j}^{11}, K_{i j}^{12}$, and $K_{i j}^{13}$ are constant coefficients for the linear terms; $C_{i j k}$ and $K_{i j k}$ are constant coefficients for the quadratic geometrical nonlinear terms; $M_{i j k l}, C_{i j k l}$, and $K_{i j k l}$ are constant coefficients for the cubic geometrical nonlinear terms; $f_{i}$ are amplitudes of the harmonic forcing terms; and $C_{i j}$ are constant coefficient of a damping term that has been introduced via a structural damping ratio $\xi$. All the coefficients in (27) can be seen in Appendix A. The trial functions in terms of the dimensionless variable $s$ are

$$
\psi_{j}(s)=\frac{1}{\sqrt{2}} \sin \left[\left(j-\frac{1}{2}\right) \pi s\right] .
$$

\section{IHB Method for Determining Periodic Responses}

Among the solution methods of ordinary differential equations, the IHB method is relatively efficient and reliable for treating the vibrations of nonlinear systems. The method has been developed and successfully applied to analyze periodic nonlinear structural vibrations. With the new dimensionless time variable $\tau$ defined as

$$
\tau=\omega t
$$


where $\omega$ is the unknown fundamental frequency of the steady-state response, and this paper assumes that the rotating angular velocity can be $\Omega=\widehat{p} \omega$, in which $\widehat{p}$ is the positive integer introduced to analyze possible subharmonic responses of the nonlinear system. Equation (27) becomes

$$
\begin{aligned}
& \omega^{2} \sum_{j=1}^{n}\left(M_{i j}+\sum_{k=1}^{n} \sum_{l=1}^{n} M_{i j k l} q_{k} q_{l}\right) q_{j}^{\prime \prime} \\
& +\omega \sum_{j=1}^{n}\left(C_{i j}+\widehat{p} \omega \sum_{k=1}^{n} C_{i j k} q_{k}+\omega \sum_{k=1}^{n} \sum_{l=1}^{n} C_{i j k l} q_{k} q_{l}^{\prime}\right) q_{j}^{\prime} \\
& \quad+\sum_{j=1}^{n}\left(K_{i j}^{11}+g \sin \widehat{p} \tau K_{i j}^{12}+\widehat{p}^{2} \omega^{2} K_{i j}^{13}\right) q_{j} \\
& +g \cos \widehat{p} \tau \sum_{j=1}^{n} \sum_{k=1}^{n} K_{i j k} q_{k} q_{j} \\
& +\hat{p}^{2} \omega^{2} \sum_{j=1}^{n} \sum_{k=1}^{n} \sum_{l=1}^{n} K_{i j k l} q_{k} q_{l} q_{j}=g f_{i} \cos \hat{p} \tau,
\end{aligned}
$$

where $q^{\prime}$ and $q^{\prime \prime}$ respect the first and second differentiation of $q$ with respect to $\tau$, respectively.

The IHB method consists of two steps; the first step of the IHB method is an incremental process, which is a NewtonRaphson procedure. Let $q_{j 0}$ and $\omega_{0}$ denote the solutions of the equation, where $j=1,2, \ldots, n$; a neighboring state can be expressed by adding increments to $q_{j 0}$ and $\omega_{0}$

$$
\begin{gathered}
q_{j}=q_{j 0}+\Delta q_{j}, \\
\omega=\omega_{0}+\Delta \omega .
\end{gathered}
$$

Substituting (31) into (30) and neglecting higher-order terms of the increments yield the following linearized incremental equation with $\Delta q_{j}$ and $\Delta \omega$ as the unknown quantity:

$$
\begin{aligned}
& \omega_{0}^{2} \sum_{j=1}^{n}\left(M_{i j}+\sum_{k=1}^{n} \sum_{l=1}^{n} M_{i j k l} q_{k 0} q_{l 0}\right) \Delta q_{j}^{\prime \prime}+\sum_{j=1}^{n}\left[\omega_{0} C_{i j}\right. \\
& \left.+\widehat{p} \omega_{0}^{2} \sum_{k=1}^{n} C_{i j k} q_{k 0}+\omega_{0}^{2} \sum_{k=1}^{n} \sum_{l=1}^{n}\left(C_{i j k l}+C_{i l k j}\right) q_{k 0} q_{l 0}^{\prime}\right] \\
& \cdot \Delta q_{j}^{\prime}+\sum_{j=1}^{n}\left[\left(K_{i j}^{11}+g \sin \hat{p} \tau K_{i j}^{12}+\widehat{p}^{2} \omega_{0}^{2} K_{i j}^{13}\right)+g\right. \\
& \cdot \cos \widehat{p} \tau \sum_{k=1}^{n}\left(K_{i j k}+K_{i k j}\right) q_{k 0} \\
& +\hat{p}^{2} \omega_{0}^{2} \sum_{k=1}^{n} \sum_{l=1}^{n}\left(K_{i j k l}+K_{i l k j}+K_{i k j l}\right) \cdot q_{k 0} q_{l 0} \\
& +\widehat{p} \omega_{0}^{2} \sum_{k=1}^{n} C_{i k j} q_{k 0}^{\prime}+\omega_{0}^{2} \sum_{k=1}^{n} \sum_{l=1}^{n}\left(M_{i k j l}+M_{i k l j}\right) q_{k 0}^{\prime \prime} q_{l 0} \\
& \left.+\omega_{0}^{2} \sum_{k=1}^{n} \sum_{l=1}^{n} C_{i k j l} q_{k 0}^{\prime} q_{l 0}^{\prime}\right] \Delta q_{j}=R_{i}
\end{aligned}
$$

$$
\begin{aligned}
& -\Delta \omega \sum_{j=1}^{n}\left[2 \omega_{0}\left(M_{i j}+\sum_{k=1}^{n} \sum_{l=1}^{n} M_{i j k l} q_{k 0} q_{l 0}\right) q_{j 0}^{\prime \prime}\right. \\
& +\left(C_{i j}+2 \widehat{p} \omega_{0} \sum_{k=1}^{n} C_{i j k} q_{k 0}+2 \omega_{0} \sum_{k=1}^{n} \sum_{l=1}^{n} C_{i j k l} q_{k 0} q_{l 0}^{\prime}\right) \\
& \cdot q_{j 0}^{\prime}+\left(2 \widehat{p}^{2} \omega_{0} K_{i j}^{13}+2 \widehat{p}^{2} \omega_{0} \sum_{k=1}^{n} \sum_{l=1}^{n} K_{i j k l} q_{k 0} q_{l 0}\right) \\
& \left.\cdot q_{j 0}\right],
\end{aligned}
$$

where

$$
\begin{aligned}
R_{i} & =g f_{i} \cos \hat{p} \tau-\left[\omega_{0}^{2} \sum_{j=1}^{n}\left(M_{i j}+\sum_{k=1}^{n} \sum_{l=1}^{n} M_{i j k l} q_{k 0} q_{l 0}\right)\right. \\
& \cdot q_{j 0}^{\prime \prime}+\omega_{0} \sum_{j=1}^{n}\left(C_{i j}+\widehat{p} \omega_{0} \sum_{k=1}^{n} C_{i j k} q_{k 0}\right. \\
& \left.+\omega_{0} \sum_{k=1}^{n} \sum_{l=1}^{n} C_{i j k l} q_{k 0} q_{l 0}^{\prime}\right) q_{j 0}^{\prime}+\sum_{j=1}^{n}\left(K_{i j}^{11}+g \sin \hat{p} \tau K_{i j}^{12}\right. \\
& \left.+\widehat{p}^{2} \omega_{0}^{2} K_{i j}^{13}\right) q_{j 0}+g \cos \widehat{p} \tau \sum_{j=1}^{n} \sum_{k=1}^{n} K_{i j k} q_{k 0} q_{j 0} \\
& \left.+\hat{p}^{2} \omega_{0}^{2} \sum_{j=1}^{n} \sum_{k=1}^{n} \sum_{l=1}^{n} K_{i j k l} q_{k 0} q_{l 0} q_{j 0}\right]
\end{aligned}
$$

is a correction term which goes to zero when the solution is exact. Equations (32) and (33) can be written in the matrix form

$$
\begin{aligned}
\omega_{0}^{2} & \left(\mathbf{M}^{11}+\mathbf{M}^{31}\right) \Delta \mathbf{q}^{\prime \prime}+\omega_{0}\left(\mathbf{C}^{11}+\widehat{p} \omega_{0} \mathbf{C}^{21}+\omega_{0} \mathbf{C}^{31}\right) \\
+ & \Delta \mathbf{q}^{\prime}+\left(\mathbf{K}^{11}+g \sin \hat{p} \tau \mathbf{K}^{12}+\widehat{p}^{2} \omega_{0}^{2} \mathbf{K}^{13}\right. \\
+ & g \cos \widehat{p} \tau \mathbf{K}^{21}+\widehat{p}^{2} \omega_{0}^{2} \mathbf{K}^{31}+\widehat{p} \omega_{0}^{2} \mathbf{C}^{22}+\omega_{0}^{2} \mathbf{M}^{32} \\
+ & \left.\omega_{0}^{2} \mathbf{C}^{32}\right) \Delta \mathbf{q}=\mathbf{R}-\Delta \omega\left[2 \omega_{0}\left(\mathbf{M}^{11}+\mathbf{M}^{31}\right) \mathbf{q}_{0}^{\prime \prime}\right. \\
+ & \left(\mathbf{C}^{11}+2 \widehat{p} \omega_{0} \mathbf{C}^{21}+2 \omega_{0} \mathbf{C}^{33}\right) \mathbf{q}_{0}^{\prime} \\
+ & \left.2 \widehat{p}^{2} \omega_{0}\left(\mathbf{K}^{13}+\mathbf{K}^{32}\right) \mathbf{q}_{0}\right],
\end{aligned}
$$

where

$$
\begin{aligned}
\mathbf{R} & =g \mathbf{F} \cos \hat{p} \tau-\left[\omega_{0}^{2}\left(\mathbf{M}^{11}+\mathbf{M}^{31}\right) \mathbf{q}_{0}^{\prime \prime}+\omega_{0}\left(\mathbf{C}^{11}\right.\right. \\
& \left.+\widehat{p} \omega_{0} \mathbf{C}^{21}+\omega_{0} \mathbf{C}^{33}\right) \mathbf{q}_{0}^{\prime}+\left(\mathbf{K}^{11}+g \sin \widehat{p} \tau \mathbf{K}^{12}\right. \\
& \left.\left.+\widehat{p}^{2} \omega_{0}^{2} \mathbf{K}^{13}+g \cos \hat{p} \tau \mathbf{K}^{22}+\widehat{p}^{2} \omega_{0}^{2} \mathbf{K}^{32}\right) \mathbf{q}_{0}\right]
\end{aligned}
$$


is the correction vector, $\mathbf{M}^{11}, \mathbf{C}^{11}, \mathbf{K}^{11}, \mathbf{K}^{12}, \mathbf{K}^{13}, \mathbf{F}, \mathbf{q}_{0}$, and $\Delta \mathbf{q}$ have entries $M_{i j}, C_{i j}, K_{i j}^{11}, K_{i j}^{12}, K_{i j}^{13}, f_{i}, q_{i 0}$, and $\Delta q_{i}$, respectively; and

$$
\begin{aligned}
& M_{i j}^{31}=\sum_{k=1}^{n} \sum_{l=1}^{n} M_{i j k l} q_{k 0} q_{l 0}, \\
& M_{i j}^{32}=\sum_{k=1}^{n} \sum_{l=1}^{n}\left(M_{i k j l}+M_{i k l j}\right) q_{k 0}^{\prime \prime} q_{l 0} \\
& C_{i j}^{21}=\sum_{k=1}^{n} C_{i j k} q_{k 0} \\
& C_{i j}^{22}=\sum_{k=1}^{n} C_{i k j} q_{k 0}^{\prime} \\
& C_{i j}^{31}=\sum_{k=1}^{n} \sum_{l=1}^{n}\left(C_{i j k l}+C_{i l k j}\right) q_{k 0} q_{l 0}^{\prime}, \\
& C_{i j}^{32}=\sum_{k=1}^{n} \sum_{l=1}^{n} C_{i k j l} q_{k 0}^{\prime} q_{l 0}^{\prime}, \\
& C_{i j}^{33}=\sum_{k=1}^{n} \sum_{l=1}^{n} C_{i j k l} q_{k 0} q_{l 0}^{\prime}, \\
& K_{i j}^{21}=\sum_{k=1}^{n}\left(K_{i j k}+K_{i k j}\right) q_{k 0},
\end{aligned}
$$

$$
\begin{aligned}
& K_{i j}^{22}=\sum_{k=1}^{n} K_{i j k} q_{k 0}, \\
& K_{i j}^{31}=\sum_{k=1}^{n} \sum_{l=1}^{n}\left(K_{i j k l}+K_{i l k j}+K_{i k j l}\right) q_{k 0} q_{l 0}, \\
& K_{i j}^{32}=\sum_{k=1}^{n} \sum_{l=1}^{n} K_{i j k l} q_{k 0} q_{l 0} .
\end{aligned}
$$

The second step of the IHB method is a harmonic balance procedure; one assumes $q_{j 0}$ and $\Delta q_{j}$ can be expressed by expanding them in truncated Fourier series

$$
\begin{aligned}
q_{j 0} & =\sum_{m=1}^{n_{c}} a_{j m} \cos (m-1) \tau+\sum_{m=1}^{n_{s}} b_{j m} \sin m \tau=\mathbf{C}_{s} \mathbf{A}_{j} \\
\Delta q_{j} & =\sum_{m=1}^{n_{c}} \Delta a_{j m} \cos (m-1) \tau+\sum_{m=1}^{n_{s}} \Delta b_{j m} \sin m \tau \\
& =\mathbf{C}_{s} \Delta \mathbf{A}_{j}
\end{aligned}
$$

where $\mathbf{C}_{s}$ is the transformation matrix, it consists of trigonometric functions, $\mathbf{A}_{j}$ is the harmonic term coefficient matrix. $\mathbf{C}_{s}, \mathbf{A}_{j}$, and $\Delta \mathbf{A}_{j}$ can be written as

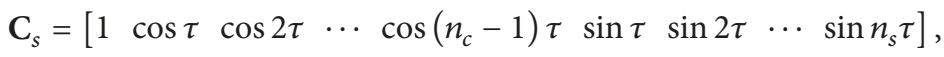

$$
\begin{aligned}
& \mathbf{A}_{j}=\left[\begin{array}{llllllll}
a_{j 1} & a_{j 2} & \cdots & a_{j n_{c}} & b_{j 1} & b_{j 2} & \cdots & b_{j n_{s}}
\end{array}\right]^{T}, \\
& \Delta \mathbf{A}_{j}=\left[\begin{array}{llllllll}
\Delta a_{j 1} & \Delta a_{j 2} & \cdots & \Delta a_{j n_{c}} & \Delta b_{j 1} & \Delta b_{j 2} & \cdots & \Delta b_{j n_{s}}
\end{array}\right]^{T}
\end{aligned}
$$

in which $a_{j m}$ and $b_{j m}$ in (37) are Fourier coefficients and $n_{c}$ and $n_{s}$ are numbers of cosine and sine harmonic terms reserved, respectively.

Hence, vectors of generalized coordinates and their increments can be written as

$$
\begin{aligned}
\mathbf{q}_{0} & =\mathbf{S A}, \\
\Delta \mathbf{q} & =\mathbf{S} \Delta \mathbf{A},
\end{aligned}
$$

where $\mathbf{S}=\operatorname{diag}\left(\mathbf{C}_{s}, \mathbf{C}_{s}, \ldots, \mathbf{C}_{s}\right), \mathbf{A}=\left[\begin{array}{llll}\mathbf{A}_{1} & \mathbf{A}_{2} & \cdots & \mathbf{A}_{n}\end{array}\right]^{T}$, and $\Delta \mathbf{A}=\left[\begin{array}{llll}\Delta \mathbf{A}_{1} & \Delta \mathbf{A}_{2} & \cdots & \Delta \mathbf{A}_{n}\end{array}\right]^{T} ;$ diag represents extracting only diagonal items in matrix $\mathbf{S}$.

Differentiating (40) yields

$$
\begin{aligned}
\mathbf{q}_{0}^{\prime} & =\mathbf{S}^{\prime} \mathbf{A}, \\
\Delta \mathbf{q}^{\prime} & =\mathbf{S}^{\prime} \Delta \mathbf{A}, \\
\mathbf{q}_{0}^{\prime \prime} & =\mathbf{S}^{\prime \prime} \mathbf{A},
\end{aligned}
$$

$$
\Delta \mathbf{q}^{\prime \prime}=\mathbf{S}^{\prime \prime} \Delta \mathbf{A}
$$

Using Galerkin procedure to balance harmonics in (34) yields

$$
\begin{aligned}
\int_{0}^{2 \pi} & \delta(\Delta \mathbf{q})^{T}\left[\omega_{0}^{2}\left(\mathbf{M}^{11}+\mathbf{M}^{31}\right) \Delta \mathbf{q}^{\prime \prime}+\omega_{0}\left(\mathbf{C}^{11}\right.\right. \\
+ & \left.\widehat{p} \omega_{0} \mathbf{C}^{21}+\omega_{0} \mathbf{C}^{31}\right) \Delta \mathbf{q}^{\prime}+\left(\mathbf{K}^{11}+g \sin \hat{p} \tau \mathbf{K}^{12}\right. \\
+ & \widehat{p}^{2} \omega_{0}^{2} \mathbf{K}^{13}+g \cos \hat{p} \tau \mathbf{K}^{21}+\widehat{p}^{2} \omega_{0}^{2} \mathbf{K}^{31}+\widehat{p} \omega_{0}^{2} \mathbf{C}^{22} \\
+ & \left.\left.\omega_{0}^{2} \mathbf{M}^{32}+\omega_{0}^{2} \mathbf{C}^{32}\right) \Delta \mathbf{q}\right] d \tau=\int_{0}^{2 \pi} \delta(\Delta \mathbf{q})^{T} \\
& \cdot\left\{\mathbf{R}-\Delta \omega\left[2 \omega_{0}\left(\mathbf{M}^{11}+\mathbf{M}^{31}\right) \mathbf{q}_{0}^{\prime \prime}\right.\right. \\
& +\left(\mathbf{C}^{11}+2 \widehat{p} \omega_{0} \mathbf{C}^{21}+2 \omega_{0} \mathbf{C}^{33}\right) \mathbf{q}_{0}^{\prime} \\
& \left.\left.+2 \widehat{p}^{2} \omega_{0}\left(\mathbf{K}^{13}+\mathbf{K}^{32}\right) \mathbf{q}_{0}\right]\right\} d \tau .
\end{aligned}
$$


Substituting (40) and (41) into (42) yields a series linear equations with $\Delta \mathbf{A}$ and $\Delta \omega$ as the unknown quantities

$$
\mathbf{K}_{A} \Delta \mathbf{A}=\mathbf{R}-\mathbf{R}_{\omega} \Delta \omega,
$$

where

$$
\begin{aligned}
\mathbf{K}_{A} & =\omega_{0}^{2}\left(\overline{\mathbf{M}}^{11}+\overline{\mathbf{M}}^{31}\right)+\omega_{0}\left(\overline{\mathbf{C}}^{11}+\widehat{p} \omega_{0} \overline{\mathbf{C}}^{21}\right. \\
& \left.+\omega_{0} \overline{\mathbf{C}}^{31}\right)+\left(\overline{\mathbf{K}}^{11}+\overline{\mathbf{K}}^{12}+\overline{\mathbf{K}}^{21}\right)+\omega_{0}^{2}\left(\widehat{p}^{2} \overline{\mathbf{K}}^{13}\right. \\
& \left.+\widehat{p}^{2} \overline{\mathbf{K}}^{31}+\widehat{p}^{22}+\overline{\mathbf{M}}^{32}+\overline{\mathbf{C}}^{32}\right), \\
\mathbf{R} & =\overline{\mathbf{F}}-\left[\omega_{0}^{2}\left(\overline{\mathbf{M}}^{11}+\overline{\mathbf{M}}^{31}\right)\right. \\
& +\omega_{0}\left(\overline{\mathbf{C}}^{11}+\widehat{p} \omega_{0} \overline{\mathbf{C}}^{21}+\omega_{0} \overline{\mathbf{C}}^{33}\right) \\
& \left.+\left(\overline{\mathbf{K}}^{11}+\overline{\mathbf{K}}^{12}+\overline{\mathbf{K}}^{22}\right)+\widehat{p}^{2} \omega_{0}^{2}\left(\overline{\mathbf{K}}^{13}+\overline{\mathbf{K}}^{32}\right)\right] \mathbf{A}, \\
\mathbf{R}_{\omega} & =\left[2 \omega_{0}\left(\overline{\mathbf{M}}^{11}+\overline{\mathbf{M}}^{31}\right)+\overline{\mathbf{C}}^{11}\right. \\
& \left.+2 \omega_{0}\left(\widehat{p}^{21}+\overline{\mathbf{C}}^{33}+\widehat{p}^{2} \overline{\mathbf{K}}^{13}+\widehat{p}^{2} \overline{\mathbf{K}}^{32}\right)\right] \mathbf{A}
\end{aligned}
$$

in which

$$
\begin{aligned}
& \overline{\mathbf{M}}^{11}=\int_{0}^{2 \pi} \mathbf{S}^{T} \mathbf{M}^{11} \mathbf{S}^{\prime \prime} d \tau, \\
& \overline{\mathbf{M}}^{31}=\int_{0}^{2 \pi} \mathbf{S}^{T} \mathbf{M}^{31} \mathbf{S}^{\prime \prime} d \tau, \\
& \overline{\mathbf{M}}^{32}=\int_{0}^{2 \pi} \mathbf{S}^{T} \mathbf{M}^{32} \mathbf{S} d \tau, \\
& \overline{\mathbf{C}}^{11}=\int_{0}^{2 \pi} \mathbf{S}^{T} \mathbf{C}^{11} \mathbf{S}^{\prime} d \tau, \\
& \overline{\mathbf{C}}^{21}=\int_{0}^{2 \pi} \mathbf{S}^{T} \mathbf{C}^{21} \mathbf{S}^{\prime} d \tau, \\
& \overline{\mathbf{C}}^{22}=\int_{0}^{2 \pi} \mathbf{S}^{T} \mathbf{C}^{22} \mathbf{S}^{\prime} d \tau, \\
& \overline{\mathbf{C}}^{31}=\int_{0}^{2 \pi} \mathbf{S}^{T} \mathbf{C}^{31} \mathbf{S}^{\prime} d \tau, \\
& \overline{\mathbf{C}}^{32}=\int_{0}^{2 \pi} \mathbf{S}^{T} \mathbf{C}^{32} \mathbf{S}^{\prime} d \tau, \\
& \overline{\mathbf{C}}^{33}=\int_{0}^{2 \pi} \mathbf{S}^{T} \mathbf{C}^{33} \mathbf{S}^{\prime} d \tau, \\
& \overline{\mathbf{K}}^{11}=\int_{0}^{2 \pi} \mathbf{S}^{T} \mathbf{K}^{11} \mathbf{S} d \tau, \\
& \overline{\mathbf{K}}^{12}=g \int_{0}^{2 \pi} \mathbf{S}^{T} \mathbf{K}^{12} \mathbf{S} \sin \hat{p} \tau d \tau, \\
& \overline{\mathbf{K}}^{13}=\int_{0}^{2 \pi} \mathbf{S}^{T} \mathbf{K}^{13} \mathbf{S} d \tau,
\end{aligned}
$$

$$
\begin{aligned}
\overline{\mathbf{K}}^{21} & =g \int_{0}^{2 \pi} \mathbf{S}^{T} \mathbf{K}^{21} \mathbf{S} \cos \hat{p} \tau d \tau, \\
\overline{\mathbf{K}}^{22} & =g \int_{0}^{2 \pi} \mathbf{S}^{T} \mathbf{K}^{22} \mathbf{S} \cos \hat{p} \tau d \tau, \\
\overline{\mathbf{K}}^{31} & =\int_{0}^{2 \pi} \mathbf{S}^{T} \mathbf{K}^{31} \mathbf{S} d \tau, \\
\overline{\mathbf{K}}^{32} & =\int_{0}^{2 \pi} \mathbf{S}^{T} \mathbf{K}^{32} \mathbf{S} d \tau, \\
\overline{\mathbf{F}} & =g \int_{0}^{2 \pi} \mathbf{S}^{T} \mathbf{F} \cos \hat{p} \tau d \tau .
\end{aligned}
$$

The vectors and entries of matrices in (45) are given in detail in Appendix B.

Note that the number of incremental unknowns ( $\triangle \mathbf{A}$ and $\Delta \omega)$ in (43) is one more than the number of equations; the solution process starts from a guessed solution. The nonlinear amplitude-frequency response is solved point by point by adding the small increments $\Delta \omega$ and $\Delta \mathbf{A}$ to the current solution. The Newton-Raphson iterative procedure is repeated until the convergent solution is reached. One can also choose the arc-length increment method combined with a cubic extrapolation technique in [26] to trace the solutions around some peaks in the nonlinear frequency response curve.

\section{Analysis of Buckling of the Beam}

Buckling and large deformation are classic problems in flexible link systems, especially those in vertical planes. For exploring the critical buckling load, the rotating beam with a tip mass is setting in vertical position and rotating angular velocity $\Omega=0$, as shown in Figure 2. It is well known that the critical buckling load for a fixed-free cantilever beam can be calculated as follows:

$$
F_{\mathrm{cr}}=\frac{\pi^{2} E I}{4 L^{2}} .
$$

The properties of the rotating hub-beam system with a tip mass are given with the length $L=1.1 \mathrm{~m}$; the hub radius $r_{0}=0.1 \mathrm{~m}$; the mass density $\rho=2700 \mathrm{~kg} / \mathrm{m}^{3}$; the modulus of elasticity $E=7.1 \times 10^{10} \mathrm{~N} / \mathrm{m}^{2}$; the cross-section area $A=$ $2 \times 10^{-4} \mathrm{~m}^{2}$ and the area rotary inertia $I=4.965 \times 10^{-10} \mathrm{~m}^{4}$; and the dimensionless parameter $r_{0}=0.0909$. Substituting $F_{\text {cr }}=m_{t} g$ into (25) yields the dimensionless tip mass

$$
\bar{m}_{t}=\frac{\pi^{2} E I}{4 L^{3} \rho A g}=12.3486 .
$$

This indicates that the buckling of the beam will not happen to the rotating system with the dimensionless tip mass $\bar{m}_{t}<$ 12.3486 .

\section{Results and Discussion}

6.1. Natural Frequencies of the Rotating Hub-Beam System with a Tip Mass. To investigate the bending vibration natural frequencies of the rotating beam at various rotational velocities 


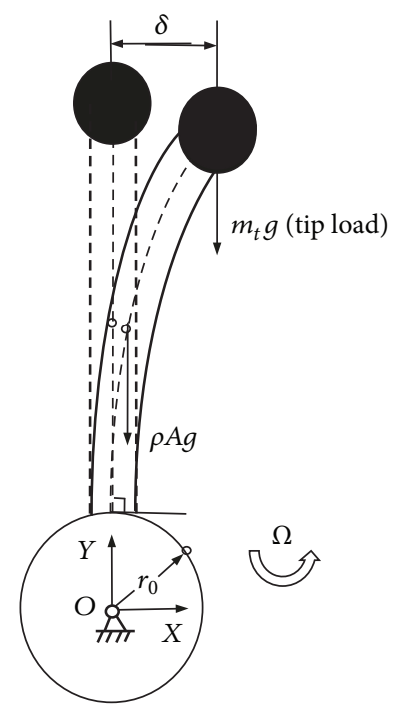

FIGURE 2: Inverted cantilevered flexible beam with a tip mass.

under different values of tip masses and hub radius ratios, natural frequencies of the rotating beam can be approximated by neglecting the nonlinear terms, the damping terms, and gravity loads in (27)

$$
\sum_{j=1}^{n} M_{i j} \ddot{q}_{j}+\sum_{j=1}^{n}\left(K_{i j}^{11}+\Omega^{2} K_{i j}^{13}\right) q_{j}=0,
$$

where $K_{i j}^{11}$ and $\Omega^{2} K_{i j}^{13}$ are the static stiffness term and the dynamic stiffness term relating to rotation of the hub, respectively. To derive the eigenvalue problem associated with (48), one assumes

$$
\mathbf{q}=e^{\tilde{j} \tilde{\omega} t} \widetilde{\boldsymbol{\Theta}}
$$

where $\tilde{j}$ is an imaginary number, $\widetilde{\omega}$ stands for a bending natural frequency, and $\widetilde{\boldsymbol{\Theta}}$ is a constant column matrix characterizing the mode shape. Substituting (49) into (48) yields

$$
\widetilde{\omega}^{2} \mathbf{M} \widetilde{\Theta}=\mathbf{K} \widetilde{\Theta},
$$

where $\mathbf{M}$ and $\mathbf{K}$ have entries $M_{i j}$ and $K_{i j}^{11}+\Omega^{2} K_{i j}^{13}$, respectively. The dimensionless natural frequency $\widetilde{\omega}$ is assessed by calculating the eigenvalues of (50).

Results of the first three dimensionless natural frequencies of the rotating hub-beam system with a tip mass from the proposed model are compared with those from [14] in Table 1. In the case of low velocity rotation, there is no need to consider the stretching of beam. And five modes are considered to reach convergence. As can be noted from Table 1, there are only small differences between the results from the two models. This indicates the use of slope angle model with five modes is sufficient to obtain right results.

It can be seen from Figure 3 that when hub radius ratio $r_{0}=0$, an increase in rotating angular velocity causes an increase in the first four natural frequencies for the five modes of vibration under different values of tip masses. Figure 3(a) shows that an increase in the tip mass always causes a decrease in the first natural frequency for all rotating angular velocities. In Figures 3(b)-3(d), the tip mass tends to depress the frequencies at low velocities of rotation; however, it tends to increase the frequencies at high velocities of rotation. Similar consequence was also obtained in [27].

Figure 4 shows the first four dimensionless natural frequencies veering behavior of the rotating beam associated with different hub radius ratios. It can be seen that the natural frequencies increase with the hub radius ratio and the rotating angular velocity.

6.2. Nonlinear Frequency Response of the Rotating Hub-Beam System with a Tip Mass under Gravity Loads. Consider the damped vibration of the rotating hub-beam system with a tip mass with gravity loads. The properties of the beam are chosen the same as in Section 5. With $n_{c}=9$ and $n_{s}=8$, (37) can be developed as

$$
q_{j 0}=A_{j 0}+\sum_{m=1}^{8} A_{j m} \cos \left(m \tau+\phi_{j m}\right)
$$

$$
j=1,2, \ldots, n,
$$

where

$$
\begin{aligned}
A_{j m} & =\sqrt{a_{j m}^{2}+b_{j m}^{2}}, \\
\phi_{j m} & =\operatorname{atan} 2\left(-b_{j m}, a_{j m}\right) .
\end{aligned}
$$

The nonlinear frequency responses of the system with a tip mass $\Omega \sim A_{11}$ and $\Omega \sim A_{21}$ when using different numbers of included trial functions are shown in Figures 5(a) and 5(b), respectively, where $A_{11}$ and $A_{21}$ are the amplitudes of the first harmonic terms of the first two generalized coordinates $q_{1}$ and $q_{2}$, respectively, and $\Omega$ is the dimensionless excitation frequency. Subharmonic responses corresponding to $\widehat{p}>1$ are not found in the frequency range shown with the numbers of included trial functions. The higher harmonics are small and not shown here. Comparison of various included trial functions for nonlinear frequency responses indicates that convergence is reached when $n=5$. Therefore, it verifies that five modes used in this work are sufficient to obtain convergent results. Due to the quadratic nonlinearities, the response of the system with a tip mass exhibits a typical softening-spring nonlinear characteristic.

6.3. Five-Mode Model for Bending Vibration Analysis with Consideration of Variation in Tip Mass. Consider the damped vibration of the rotating hub-beam system with a tip mass with respect to the variation of tip mass: $g=0.2, \xi=0.01$, and $r_{0}=0.0909$. In Section 6.2, it is indicated that a fivemode model is efficient and accurate to calculate nonlinear frequency response. In all cases below, $n=5, n_{c}=9$, and $n_{s}=8$ in (37) are used. The tip deformation of the rotating beam is given by

$$
w(L, t)=\int_{0}^{L} \phi(s, t) d s .
$$




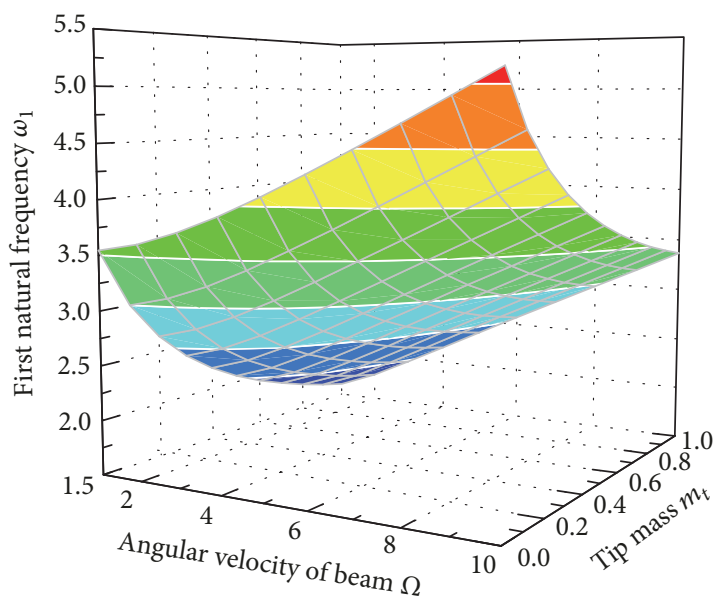

(a)

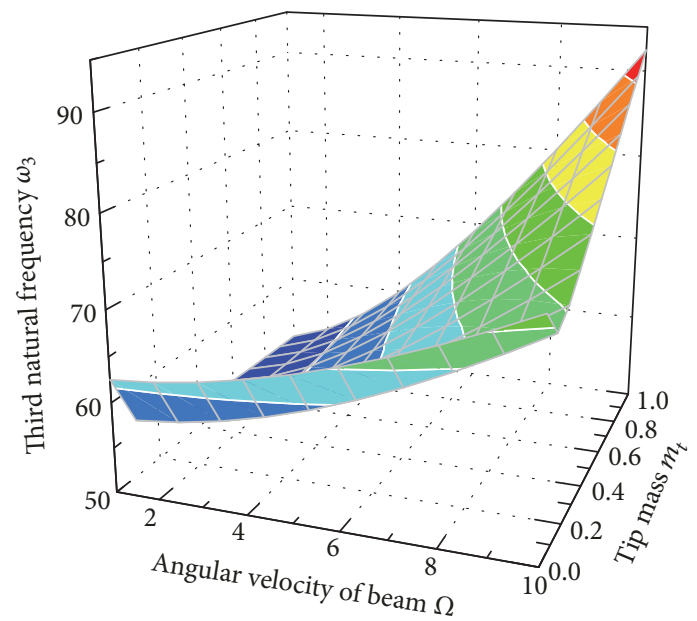

(c)

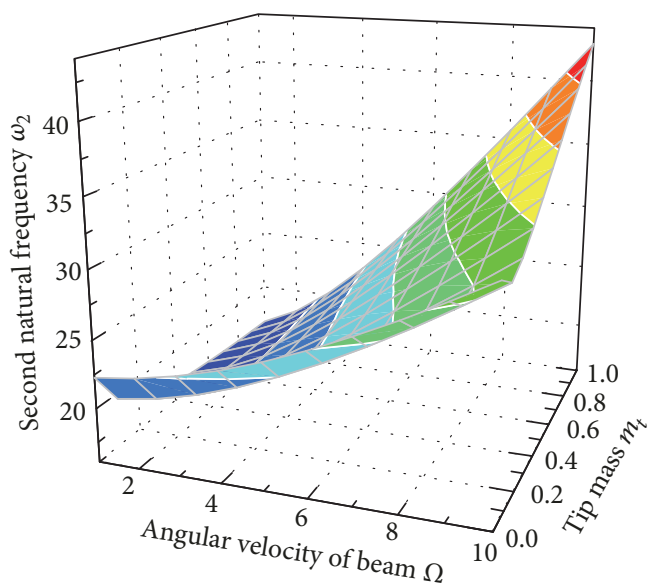

(b)

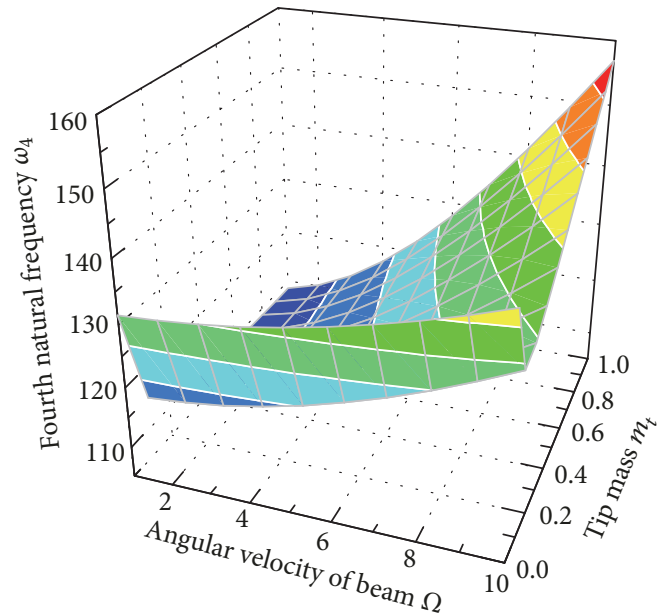

(d)

FiguRE 3: Variations of first four dimensionless natural frequencies $\omega_{i}$ as functions of rotating angular velocity $\Omega$ and tip mass $m_{t}$ for hub radius ratio $r_{0}=0$ : (a) first natural frequency $\omega_{1}$; (b) second natural frequency $\omega_{2}$; (c) third natural frequency $\omega_{3}$; and (d) fourth natural frequency $\omega_{4}$.

TABLE 1: Comparison of the first three dimensionless natural frequencies of the rotating hub-beam system with a tip mass in [14] for $r_{0}=0$ and $\Omega=0$.

\begin{tabular}{|c|c|c|c|c|c|c|}
\hline \multicolumn{3}{|c|}{ First natural frequency $\omega_{1}$} & \multicolumn{2}{|c|}{ Second natural frequency $\omega_{2}$} & \multicolumn{2}{|c|}{ Third natural frequency $\omega_{3}$} \\
\hline$m_{t}$ & Reference [14] & $\begin{array}{c}\text { Slope angle } \\
\text { model }(n=5)\end{array}$ & Reference [14] & $\begin{array}{c}\text { Slope angle } \\
\text { model }(n=5)\end{array}$ & Reference [14] & $\begin{array}{c}\text { Slope angle } \\
\text { model }(n=5)\end{array}$ \\
\hline 0 & 3.516 & 3.517 & 22.035 & 22.082 & 61.697 & 62.289 \\
\hline 1 & 1.557 & 1.557 & 16.250 & 16.277 & 50.896 & 51.179 \\
\hline 2 & 1.158 & 1.158 & 15.861 & 15.887 & 50.448 & 50.722 \\
\hline 3 & 0.963 & 0.963 & 15.720 & 15.746 & 50.291 & 50.561 \\
\hline 4 & 0.842 & 0.842 & 15.647 & 15.673 & 50.211 & 50.478 \\
\hline
\end{tabular}

Figure 6 shows the effect of tip mass on tip amplitude of the rotating beam $\Omega \sim A_{L}$, where the tip amplitude of the beam is defined as $A_{L}=\{\max [w(L, t)]-\min [w(L, t)]\} / 2$. It can be seen that the tip amplitude of the rotating flexible beam gets larger with increasing the tip mass. Moreover, it can be seen that the region which resonant behavior appears tends to be located in the lower frequency range as the tip mass increases. Resonant behavior can be produced when the system is excited at a frequency close to the natural frequency. The system's natural frequency strongly depends on the tip mass.

The comparison of bending vibration analysis between the gravity of beam considered and ignored model is also presented in Figure 6. It can be seen that discrepancies of results 




(a)

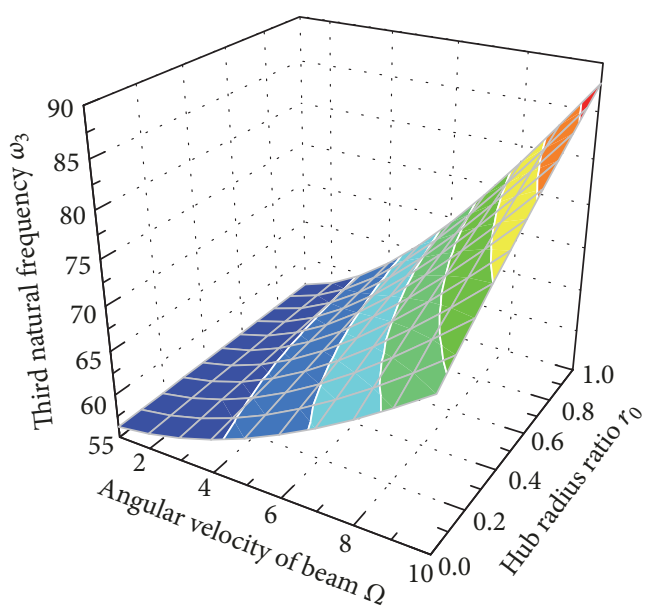

(c)

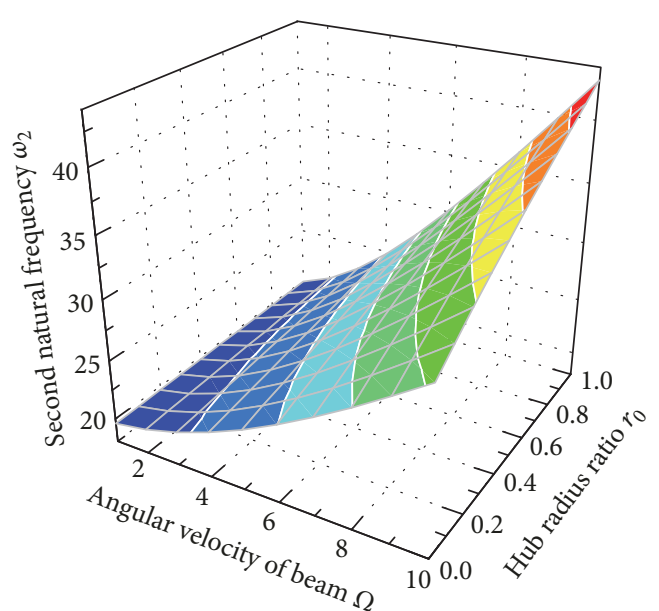

(b)

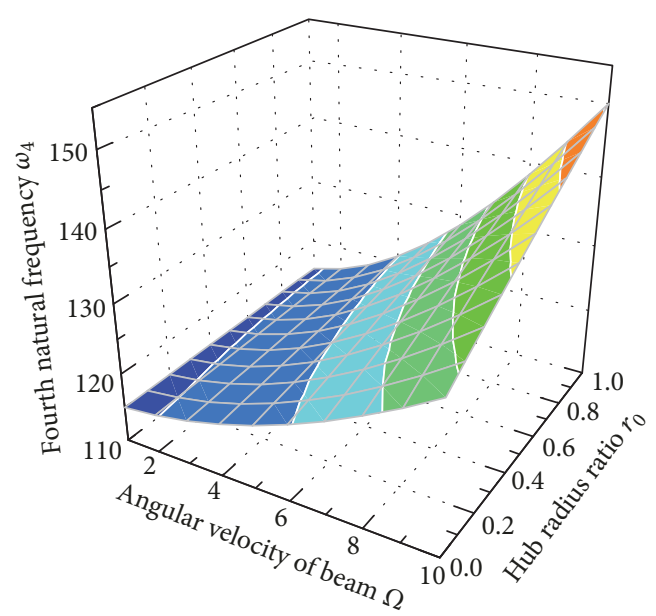

(d)

FIGURE 4: Variations of first four dimensionless natural frequencies $\omega_{i}$ as functions of rotating angular velocity $\Omega$ and hub radius ratio $r_{0}$ for tip mass $m_{t}=0.1$ : (a) first natural frequency $\omega_{1}$; (b) second natural frequency $\omega_{2}$; (c) third natural frequency $\omega_{3}$; and (d) fourth natural frequency $\omega_{4}$.

from the two models are remarkable, and tip amplitude calculated from the gravity of beam ignored model is much smaller than that from the gravity of beam considered model. This indicates that consideration of taking into account the gravity of beam in the model of rotating hub-beam system with a tip mass is necessary.

6.4. Five-Mode Model for Bending Vibration Analysis with Consideration of Variation in Damping Ratio. Figure 7 shows nonlinear frequency response curves of the rotating flexible beam $\Omega \sim A_{L}$ for different values of damping ratios with $g=0.2, m_{t}=0.05$, and $r_{0}=0.0909$. As shown in Figure 7, the tip amplitude of the rotating flexible beam decreases as damping ratio increases. It indicates that damping ratio plays an important role to decrease the tip amplitude and enhance the stabilizing effect on the system. In addition, the tip amplitudes of the rotating flexible beam have the same region which resonant behavior appears for all of damping ratios, and the tip amplitudes are small and almost identical when excitation frequency is far separated from the natural frequency.

\section{Conclusions}

The dynamic characteristics of a flexible hub-beam system with a tip mass have been investigated in this work. The motion of the beam with a tip mass is described based on the slope angle model, and gravity loads of beam and tip mass introduce combined parametric and forced harmonic excitations in the resulting equations of motion. The IHB method is used to solve for periodic responses of a fivedimensional model of the rotating hub-beam system with a tip mass for which convergence is reached.

The buckling will not happen to the rotating beam if the value of tip mass within the critical buckling load range. Natural frequencies of the rotating hub-beam system with a tip mass calculated using the slope angle model with five assumed modes show good agreement with those in [14]. 


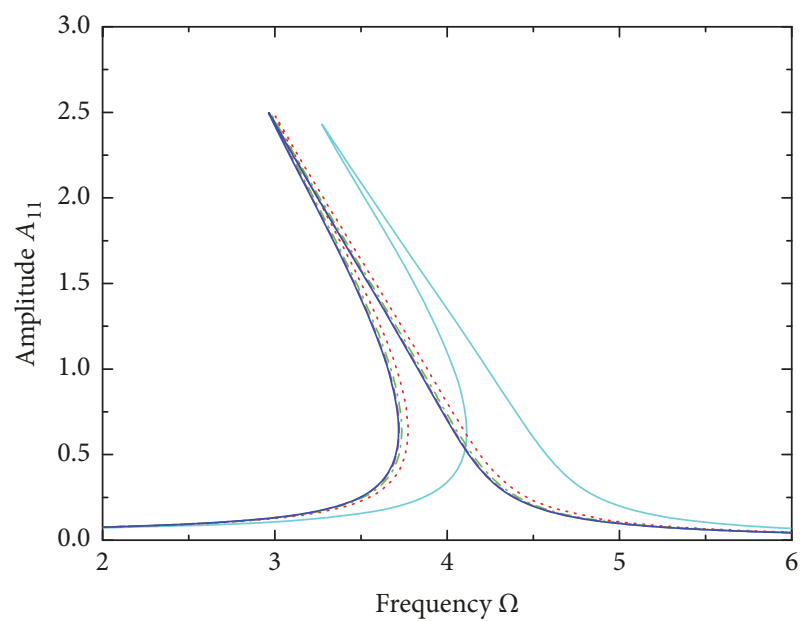

$\begin{array}{ll}\text { One assumed mode } & --- \text { Four assumed modes } \\ \ldots . . . & \text { Two assumed modes } \quad \_ \text {Five assumed modes } \\ \ldots & \text { Three assumed modes }\end{array}$

(a)

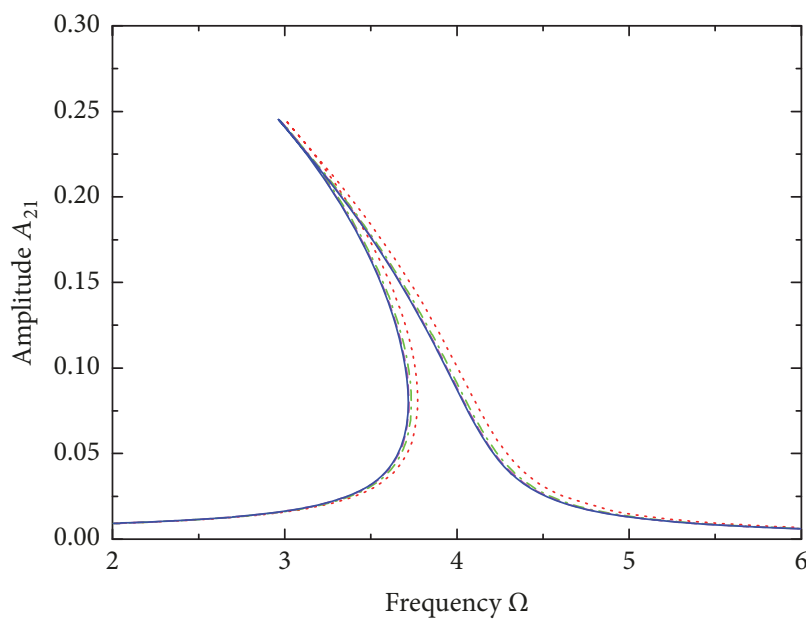

..... Two assumed modes $\quad$ - - - Four assumed modes -... Three assumed modes _ _ Five assumed modes

(b)

FIGURE 5: Nonlinear frequency response curves of the rotating hub-beam system with a tip mass for $g=0.2, \xi=0.01, r_{0}=0.0909$, and $m_{t}=0.035$, calculated using different numbers of included trial functions in (28): (a) $\Omega \sim A_{11}$; (b) $\Omega \sim A_{21}$.

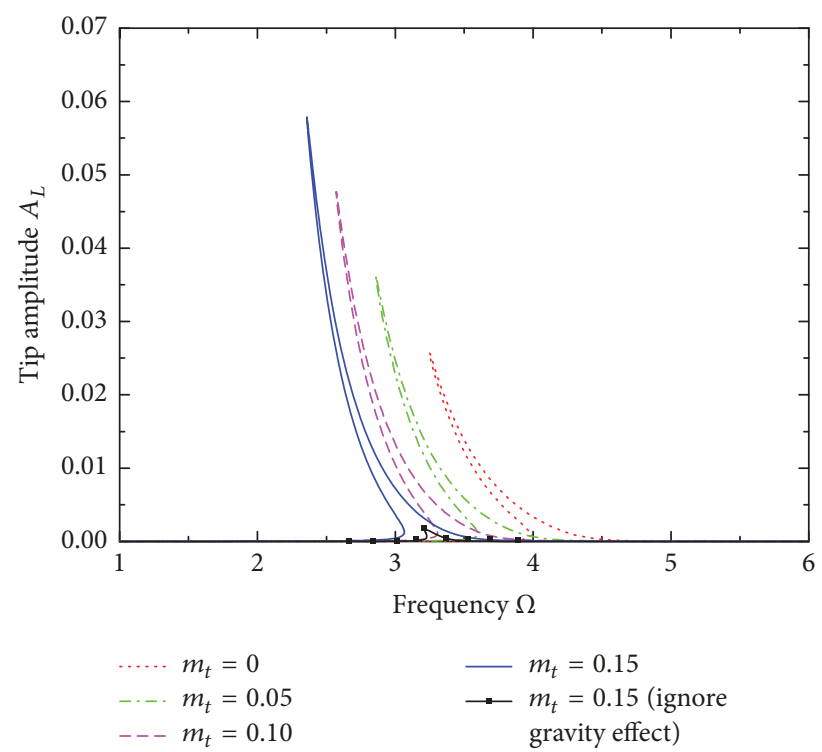

FIGURE 6: Tip amplitude diagram with respect to the variation in tip mass $m_{t}$ for $g=0.2, \xi=0.01, r_{0}=0.0909$, and $n=5$.

Comparisons of the nonlinear frequency response curves under different values of tip masses and damping ratios are obtained by using the IHB method. The nonlinear frequency response curves show that the tip amplitude of the rotating beam increases with the tip mass, while the tip amplitude of that decreases with damping ratio. On the other hand, the frequency response curves show different structures of resonance regions according to the values of the tip mass and damping ratio. In addition, large discrepancy exists between the results obtained from the gravity of beam considered and those from the gravity of beam ignored.

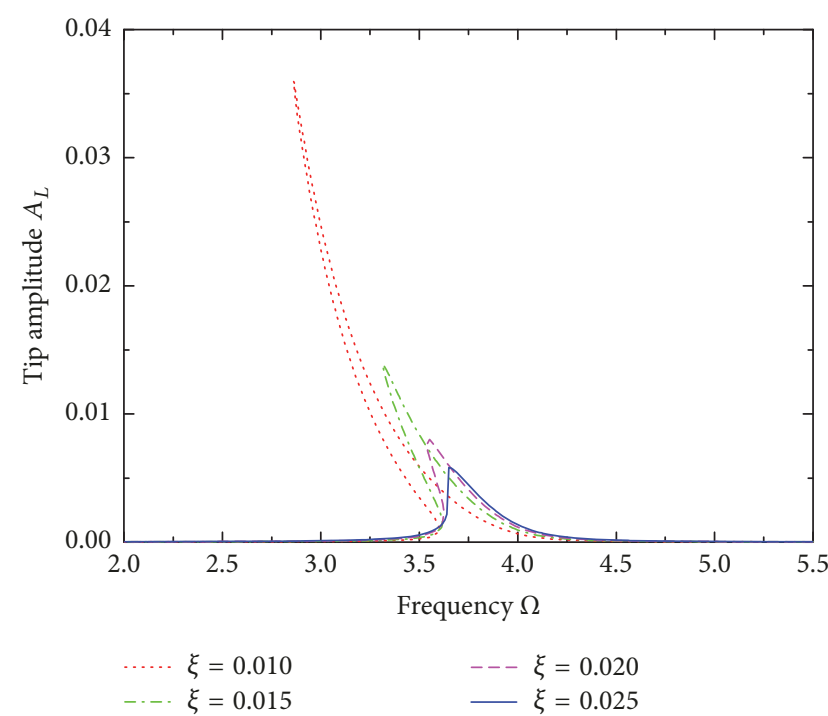

FIGURE 7: Tip amplitude diagram with respect to the variation in damping ratio $\xi$ for $g=0.2, m_{t}=0.05, r_{0}=0.0909$, and $n=5$.

\section{Appendix}

\section{A. Coefficients in the Spatially Discretized Equations in (27)}

The coefficients in (27) about the linear, quadratic nonlinear, cubic nonlinear, damping, and excitation force terms are written as

$$
M_{i j}=\int_{0}^{1} \int_{0}^{s} \psi_{i}(\tau) d \tau \int_{0}^{s} \psi_{j}(\tau) d \tau d s
$$


Shock and Vibration

13

$$
\begin{array}{rlrl}
+ & m_{t} \int_{0}^{1} \psi_{i}(s) d s \int_{0}^{1} \psi_{j}(s) d s, & \int_{0}^{s} \psi_{j}(\tau) d \tau d s+r_{0} m_{t} \int_{0}^{1} \psi_{i}(s) \psi_{j}(s) d s \\
M_{i j k l}=\int_{0}^{1} \int_{0}^{s} \psi_{i}(\tau) \psi_{k}(\tau) d \tau \int_{0}^{s} \psi_{j}(\tau) \psi_{l}(\tau) d \tau d s & +m_{t} \int_{0}^{1} \psi_{i}(s) \psi_{j}(s) d s-m_{t} \int_{0}^{1} \psi_{i}(s) d s \\
-\frac{1}{2} \int_{0}^{1} \int_{0}^{s} \psi_{i}(\tau) d \tau \int_{0}^{s} \psi_{j}(\tau) \psi_{k}(\tau) \psi_{l}(\tau) d \tau d s & \cdot \int_{0}^{1} \psi_{j}(s) d s, \\
-\frac{1}{2} \int_{0}^{1} \int_{0}^{s} \psi_{j}(\tau) d \tau \int_{0}^{s} \psi_{i}(\tau) \psi_{k}(\tau) \psi_{l}(\tau) d \tau d s & K_{i j k}=-\frac{1}{2} \int_{0}^{1} \int_{0}^{s} \psi_{i}(\tau) \psi_{j}(\tau) \psi_{k}(\tau) d \tau d s-\frac{1}{2} \\
-\frac{1}{2} m_{t} \int_{0}^{1} \psi_{i}(s) d s \int_{0}^{1} \psi_{j}(s) \psi_{k}(\tau) \psi_{l}(\tau) d s & \cdot m_{t} \int_{0}^{1} \psi_{i}(s) \psi_{j}(s) \psi_{k}(s) d s, \\
+m_{t} \int_{0}^{1} \psi_{i}(s) \psi_{k}(\tau) d s \int_{0}^{1} \psi_{j}(s) \psi_{l}(\tau) d s-\frac{1}{2} & K_{i j k l}=-\frac{1}{2} \int_{0}^{1} \int_{0}^{s} \psi_{i}(\tau) \psi_{j}(\tau) d \tau \\
\cdot m_{t} \int_{0}^{1} \psi_{j}(s) d s \int_{0}^{1} \psi_{i}(s) \psi_{k}(\tau) \psi_{l}(\tau) d s, & \cdot \int_{0}^{s} \psi_{k}(\tau) \psi_{l}(\tau) d \tau d s-\frac{1}{2} m_{t} \int_{0}^{1} \psi_{i}(s) \\
C_{i j}=\xi \omega_{i} \int_{0}^{1} \int_{0}^{s} \psi_{i}(\tau) d \tau \int_{0}^{s} \psi_{j}(\tau) d \tau d s, & \cdot \psi_{j}(s) d s \int_{0}^{1} \psi_{k}(s) \psi_{l}(s) d s, \\
C_{i j k}=2 \int_{0}^{1} \int_{0}^{s} \psi_{i}(\tau) \psi_{k}(\tau) d \tau \int_{0}^{s} \psi_{j}(\tau) d \tau d s & f_{i}=-\int_{0}^{1} \int_{0}^{s} \psi_{i}(\tau) d \tau d s-m_{t} \int_{0}^{1} \psi_{i}(s) d s
\end{array}
$$$$
+2 m_{t} \int_{0}^{1} \psi_{j}(s) d s \int_{0}^{1} \psi_{i}(s) \psi_{k}(s) d s
$$$$
-2 m_{t} \int_{0}^{1} \psi_{i}(s) d s \int_{0}^{1} \psi_{j}(s) \psi_{k}(s) d s
$$

B. Entries of Matrices and the Vectors in (45)

Entries of matrices and the vectors in (45) are written as

$$
\begin{aligned}
& {\left[\overline{\mathbf{M}}^{11}\right]_{p, q}=-\pi M_{i j}(p-1)^{2} \delta(p, q),} \\
& p, q=1,2, \ldots, n_{c} \\
& {\left[\overline{\mathbf{M}}^{11}\right]_{p, q+n_{c}}=0, \quad p=1,2, \ldots, n_{c}, q=1,2, \ldots, n_{s},} \\
& {\left[\overline{\mathbf{M}}^{11}\right]_{p+n_{c}, q}=0, \quad p=1,2, \ldots, n_{s}, q=1,2, \ldots, n_{c},} \\
& {\left[\overline{\mathbf{M}}^{11}\right]_{p+n_{c}, q+n_{c}}=-\pi M_{i j} q^{2} \delta(p, q),} \\
& p, q=1,2, \ldots, n_{s}, \\
& {\left[\overline{\mathbf{M}}^{31}\right]_{p, q}=-\sum_{k=1}^{n} \sum_{l=1}^{n} M_{i j k l}\left[\sum_{m=1}^{n_{c}} \sum_{t=1}^{n_{c}} a_{k m}\right.} \\
& \cdot a_{l t} \alpha_{(m-1)(t-1)(p-1)(q-1)}^{(1)}+\sum_{m=1}^{n_{s}} \sum_{t=1}^{n_{s}} b_{k m} \\
& \left.\cdot b_{l t} \alpha_{m t(p-1)(q-1)}^{(3)}\right](q-1)^{2}, \quad p, q=1,2, \ldots, n_{c} \text {, } \\
& {\left[\overline{\mathbf{M}}^{31}\right]_{p, q+n_{c}}=-2 \sum_{k=1}^{n} \sum_{l=1}^{n} M_{i j k l}\left[\sum_{m=1}^{n_{c}} \sum_{t=1}^{n_{s}} a_{k m}\right.} \\
& \left.\cdot b_{l t} \alpha_{t q(m-1)(p-1)}^{(3)}\right] q^{2}
\end{aligned}
$$$$
C_{i j k l}=\int_{0}^{1} \int_{0}^{s} \psi_{i}(\tau) \psi_{k}(\tau) d \tau \int_{0}^{s} \psi_{j}(\tau) \psi_{l}(\tau) d \tau d s
$$$$
-\int_{0}^{1} \int_{0}^{s} \psi_{i}(\tau) d \tau \int_{0}^{s} \psi_{j}(\tau) \psi_{k}(\tau) \psi_{l}(\tau) d \tau d s
$$$$
+m_{t} \int_{0}^{1} \psi_{i}(s) \psi_{k}(s) d s \int_{0}^{1} \psi_{j}(s) \psi_{l}(s) d s
$$$$
-m_{t} \int_{0}^{1} \psi_{i}(s) d s \int_{0}^{1} \psi_{j}(s) \psi_{k}(s) \psi_{l}(s) d s,
$$$$
K_{i j}^{11}=\int_{0}^{1} \frac{1}{2}\left(i-\frac{1}{2}\right)\left(j-\frac{1}{2}\right) \pi^{2} \cos \left(i-\frac{1}{2}\right) \pi s
$$$$
+\int_{0}^{1} s \int_{0}^{s} \psi_{i}(\tau) \psi_{j}(\tau) d \tau d s-\int_{0}^{1} \int_{0}^{s} \psi_{i}(\tau) d \tau
$$ 
14

Shock and Vibration

$$
\begin{aligned}
& p=1,2, \ldots, n_{c}, q=1,2, \ldots, n_{s}, \\
& {\left[\overline{\mathbf{M}}^{31}\right]_{p+n_{c}, q}=-2 \sum_{k=1}^{n} \sum_{l=1}^{n} M_{i j k l}\left[\sum_{m=1}^{n_{c}} \sum_{t=1}^{n_{s}} a_{k m}\right.} \\
& \left.\cdot b_{l t} \alpha_{t p(m-1)(q-1)}^{(3)}\right](q-1)^{2} \text {, } \\
& p=1,2, \ldots, n_{s}, q=1,2, \ldots, n_{c}, \\
& {\left[\overline{\mathbf{M}}^{31}\right]_{p+n_{c}, q+n_{c}}=-\sum_{k=1}^{n} \sum_{l=1}^{n} M_{i j k l}\left[\sum_{m=1}^{n_{c}} \sum_{t=1}^{n_{c}} a_{k m}\right.} \\
& \left.\cdot a_{l t} \alpha_{p q(m-1)(t-1)}^{(3)}+\sum_{m=1}^{n_{s}} \sum_{t=1}^{n_{s}} b_{k m} b_{l t} \alpha_{m t p q}^{(2)}\right] q^{2} \text {, } \\
& p, q=1,2, \ldots, n_{s}, \\
& {\left[\overline{\mathbf{C}}^{11}\right]_{p, q+n_{c}}=\pi C_{i j} q \delta(p-1, q),} \\
& p=1,2, \ldots, n_{c}, q=1,2, \ldots, n_{s}, \\
& {\left[\overline{\mathbf{C}}^{11}\right]_{p+n_{c}, q}=\pi C_{i j}(1-q) \delta(p, q-1),} \\
& p=1,2, \ldots, n_{s}, q=1,2, \ldots, n_{c}, \\
& {\left[\overline{\mathbf{C}}^{11}\right]_{p+n_{c}, q+n_{c}}=0, \quad p, q=1,2, \ldots, n_{s},} \\
& {\left[\overline{\mathbf{C}}^{21}\right]_{p, q}=\sum_{k=1}^{n} C_{i j k} \sum_{m=1}^{n_{s}}\left[b_{k m} \beta_{(p-1) m(q-1)}^{(2)}\right](1-q),} \\
& p, q=1,2, \ldots, n_{c}, \\
& {\left[\overline{\mathbf{C}}^{21}\right]_{p, q+n_{c}}=\sum_{k=1}^{n} C_{i j k} \sum_{m=1}^{n_{c}}\left[a_{k m} \beta_{(m-1)(p-1) q}^{(1)}\right] q,} \\
& {\left[\overline{\mathbf{M}}^{32}\right]_{p, q}=-\sum_{k=1}^{n} \sum_{l=1}^{n}\left(M_{i k j l}+M_{i k l j}\right)\left[\sum_{m=1}^{n_{c}} \sum_{t=1}^{n_{c}} a_{k m}\right.} \\
& p=1,2, \ldots, n_{c}, q=1,2, \ldots, n_{s}, \\
& \cdot a_{l t}(m-1)^{2} \alpha_{(m-1)(t-1)(p-1)(q-1)}^{(1)}+\sum_{m=1}^{n_{s}} \sum_{t=1}^{n_{s}} b_{k m} \\
& {\left[\overline{\mathbf{C}}^{21}\right]_{p+n_{c}, q}=\sum_{k=1}^{n} C_{i j k} \sum_{m=1}^{n_{c}}\left[a_{k m} \beta_{(m-1) p(q-1)}^{(2)}\right](1-q) \text {, }} \\
& p=1,2, \ldots, n_{s}, q=1,2, \ldots, n_{c}, \\
& \left.\cdot b_{l t} m^{2} \alpha_{m t(p-1)(q-1)}^{(3)}\right], \quad p, q=1,2, \ldots, n_{c} \\
& {\left[\overline{\mathbf{M}}^{32}\right]_{p, q+n_{c}}=-\sum_{k=1}^{n} \sum_{l=1}^{n}\left(M_{i k j l}+M_{i k l j}\right)\left[\sum_{m=1}^{n_{c}} \sum_{t=1}^{n_{s}} a_{k m}\right.} \\
& \cdot b_{l t}(m-1)^{2} \alpha_{t q(m-1)(p-1)}^{(3)}+\sum_{m=1}^{n_{s}} \sum_{t=1}^{n_{c}} b_{k m} \\
& \left.\cdot a_{l t} m^{2} \alpha_{m q(t-1)(p-1)}^{(3)}\right] \\
& p=1,2, \ldots, n_{c}, q=1,2, \ldots, n_{s}, \\
& {\left[\overline{\mathbf{M}}^{32}\right]_{p+n_{c}, q}=-\sum_{k=1}^{n} \sum_{l=1}^{n}\left(M_{i k j l}+M_{i k l j}\right)\left[\sum_{m=1}^{n_{c}} \sum_{t=1}^{n_{s}} a_{k m}\right.} \\
& \cdot b_{l t}(m-1)^{2} \alpha_{t p(m-1)(q-1)}^{(3)}+\sum_{m=1}^{n_{s}} \sum_{t=1}^{n_{c}} b_{k m} \\
& \left.\cdot a_{l t} m^{2} \alpha_{m p(t-1)(q-1)}^{(3)}\right] \\
& p=1,2, \ldots, n_{s}, q=1,2, \ldots, n_{c}, \\
& {\left[\overline{\mathbf{M}}^{32}\right]_{p+n_{c}, q+n_{c}}=-\sum_{k=1}^{n} \sum_{l=1}^{n}\left(M_{i k j l}+M_{i k l j}\right)\left[\sum_{m=1}^{n_{c}} \sum_{t=1}^{n_{c}} a_{k m}\right.} \\
& \left.\cdot a_{l t}(m-1)^{2} \alpha_{p q(m-1)(t-1)}^{(3)}+\sum_{m=1}^{n_{s}} \sum_{t=1}^{n_{s}} b_{k m} b_{l t} m^{2} \alpha_{m t p q}^{(2)}\right] \text {, } \\
& p, q=1,2, \ldots, n_{s}, \\
& {\left[\overline{\mathbf{C}}^{11}\right]_{p, q}=0, \quad p, q=1,2, \ldots, n_{c},} \\
& {\left[\overline{\mathbf{C}}^{21}\right]_{p+n_{c}, q+n_{c}}=\sum_{k=1}^{n} C_{i j k} \sum_{m=1}^{n_{s}}\left[b_{k m} \beta_{q m p}^{(2)}\right] q \text {, }} \\
& p, q=1,2, \ldots, n_{s}, \\
& {\left[\overline{\mathbf{C}}^{22}\right]_{p, q}=\sum_{k=1}^{n} C_{i k j} \sum_{m=1}^{n_{c}}\left[a_{k m}(1-m) \beta_{(p-1)(m-1)(q-1)}^{(2)}\right]} \\
& \cdot(1-q), \quad p, q=1,2, \ldots, n_{c} \text {, } \\
& {\left[\overline{\mathbf{C}}^{22}\right]_{p, q+n_{c}}=\sum_{k=1}^{n} C_{i k j} \sum_{m=1}^{n_{s}}\left[b_{k m} m \beta_{m q(p-1)}^{(1)}\right] q \text {, }} \\
& p=1,2, \ldots, n_{c}, q=1,2, \ldots, n_{s}, \\
& {\left[\overline{\mathbf{C}}^{22}\right]_{p+n_{c}, q}=\sum_{k=1}^{n} C_{i k j} \sum_{m=1}^{n_{s}}\left[b_{k m} m \beta_{m p(q-1)}^{(2)}\right](1-q) \text {, }} \\
& p=1,2, \ldots, n_{s}, q=1,2, \ldots, n_{c}, \\
& {\left[\overline{\mathbf{C}}^{22}\right]_{p+n_{c}, q+n_{c}}=\sum_{k=1}^{n} C_{i k j} \sum_{m=1}^{n_{c}}\left[a_{k m}(1-m) \beta_{q(m-1) p}^{(2)}\right] q \text {, }} \\
& p, q=1,2, \ldots, n_{s}, \\
& {\left[\overline{\mathbf{C}}^{31}\right]_{p, q}=\sum_{k=1}^{n} \sum_{l=1}^{n}\left(C_{i j k l}+C_{i l k j}\right)\left[\sum_{m=1}^{n_{c}} \sum_{t=1}^{n_{c}} a_{k m} a_{l t}(1-t)\right.} \\
& \left.\cdot \alpha_{(t-1)(q-1)(m-1)(p-1)}^{(3)}+\sum_{m=1}^{n_{s}} \sum_{t=1}^{n_{s}} b_{k m} b_{l t} t \alpha_{m(q-1) t(p-1)}^{(3)}\right] \\
& \cdot(1-q), \quad p, q=1,2, \ldots, n_{c}, \\
& {\left[\overline{\mathbf{C}}^{31}\right]_{p, q+n_{c}}=\sum_{k=1}^{n} \sum_{l=1}^{n}\left(C_{i j k l}+C_{i l k j}\right)\left[\sum_{m=1}^{n_{c}} \sum_{t=1}^{n_{s}} a_{k m}\right.}
\end{aligned}
$$




$$
\begin{aligned}
& \cdot b_{l t} t \alpha_{(m-1) t(p-1) q}^{(1)}+\sum_{m=1}^{n_{s}} \sum_{t=1}^{n_{c}} b_{k m} a_{l t}(1-t) \\
& {\left[\overline{\mathbf{C}}^{33}\right]_{p, q}=\sum_{k=1}^{n} \sum_{l=1}^{n} C_{i j k l}\left[\sum_{m=1}^{n_{c}} \sum_{t=1}^{n_{c}} a_{k m} a_{l t}(1-t)\right.} \\
& \left.\cdot \alpha_{m(t-1)(p-1) q}^{(3)}\right] q \text {, } \\
& p=1,2, \ldots, n_{c}, q=1,2, \ldots, n_{s}, \\
& {\left[\overline{\mathbf{C}}^{31}\right]_{p+n_{c}, q}=\sum_{k=1}^{n} \sum_{l=1}^{n}\left(C_{i j k l}+C_{i l k j}\right)\left[\sum_{m=1}^{n_{c}} \sum_{t=1}^{n_{s}} a_{k m}\right.} \\
& \cdot b_{l t} t \alpha_{p(q-1)(m-1) t}^{(3)}+\sum_{m=1}^{n_{s}} \sum_{t=1}^{n_{c}} b_{k m} a_{l t}(1-t) \\
& \left.\cdot \alpha_{m(t-1) p(q-1)}^{(2)}\right](1-q) \\
& p=1,2, \ldots, n_{s}, q=1,2, \ldots, n_{c}, \\
& {\left[\overline{\mathbf{C}}^{31}\right]_{p+n_{c}, q+n_{c}}=\sum_{k=1}^{n} \sum_{l=1}^{n}\left(C_{i j k l}+C_{i l k j}\right)\left[\sum_{m=1}^{n_{c}} \sum_{t=1}^{n_{c}} a_{k m}\right.} \\
& \left.\cdot a_{l t}(1-t) \alpha_{(t-1) p(m-1) q}^{(3)}+\sum_{m=1}^{n_{s}} \sum_{t=1}^{n_{s}} b_{k m} b_{l t} t \alpha_{m p t q}^{(3)}\right] q \text {, } \\
& p, q=1,2, \ldots, n_{s}, \\
& {\left[\overline{\mathbf{C}}^{32}\right]_{p, q}=\sum_{k=1}^{n} \sum_{l=1}^{n} C_{i k j l}\left[\sum_{m=1}^{n_{c}} \sum_{t=1}^{n_{s}} a_{k m} b_{l t}(1-m)\right.} \\
& \cdot t \alpha_{(m-1)(q-1) t(p-1)}^{(3)}+\sum_{m=1}^{n_{s}} \sum_{t=1}^{n_{c}} b_{k m} a_{l t} m(1-t) \\
& \left.\cdot \alpha_{(t-1)(q-1) m(p-1)}^{(3)}\right](1-q), \quad p, q=1,2, \ldots, n_{c} \\
& {\left[\overline{\mathbf{C}}^{32}\right]_{p, q+n_{c}}=\sum_{k=1}^{n} \sum_{l=1}^{n} C_{i k j l}\left[\sum_{m=1}^{n_{c}} \sum_{t=1}^{n_{c}} a_{k m} a_{l t}(1-m)(1-t)\right.} \\
& \left.\cdot \alpha_{(m-1)(t-1)(p-1) q}^{(3)}+\sum_{m=1}^{n_{s}} \sum_{t=1}^{n_{s}} b_{k m} b_{l t} m t \alpha_{m t(p-1) q}^{(1)}\right] q \text {, } \\
& p=1,2, \ldots, n_{c}, q=1,2, \ldots, n_{s}, \\
& {\left[\overline{\mathbf{C}}^{32}\right]_{p+n_{c}, q}=\sum_{k=1}^{n} \sum_{l=1}^{n} C_{i k j l}\left[\sum_{m=1}^{n_{c}} \sum_{t=1}^{n_{c}} a_{k m} a_{l t}(1-m)(1-t)\right.} \\
& \left.\cdot \alpha_{(m-1)(t-1) p(q-1)}^{(2)}+\sum_{m=1}^{n_{s}} \sum_{t=1}^{n_{s}} b_{k m} b_{l t} m t \alpha_{p(q-1) m t}^{(3)}\right](1 \\
& \text {-q), } \quad p=1,2, \ldots, n_{s}, q=1,2, \ldots, n_{c} \text {, } \\
& {\left[\overline{\mathbf{C}}^{32}\right]_{p+n_{c}, q+n_{c}}=\sum_{k=1}^{n} \sum_{l=1}^{n} C_{i k j l}\left[\sum_{m=1}^{n_{c}} \sum_{t=1}^{n_{s}} a_{k m} b_{l t}(1-m)\right.} \\
& \left.\cdot t \alpha_{(m-1) p t q}^{(3)}+\sum_{m=1}^{n_{s}} \sum_{t=1}^{n_{c}} a_{l t} b_{k m} m(1-t) \alpha_{(t-1) p m q}^{(3)}\right] q, \\
& p, q=1,2, \ldots, n_{s},
\end{aligned}
$$

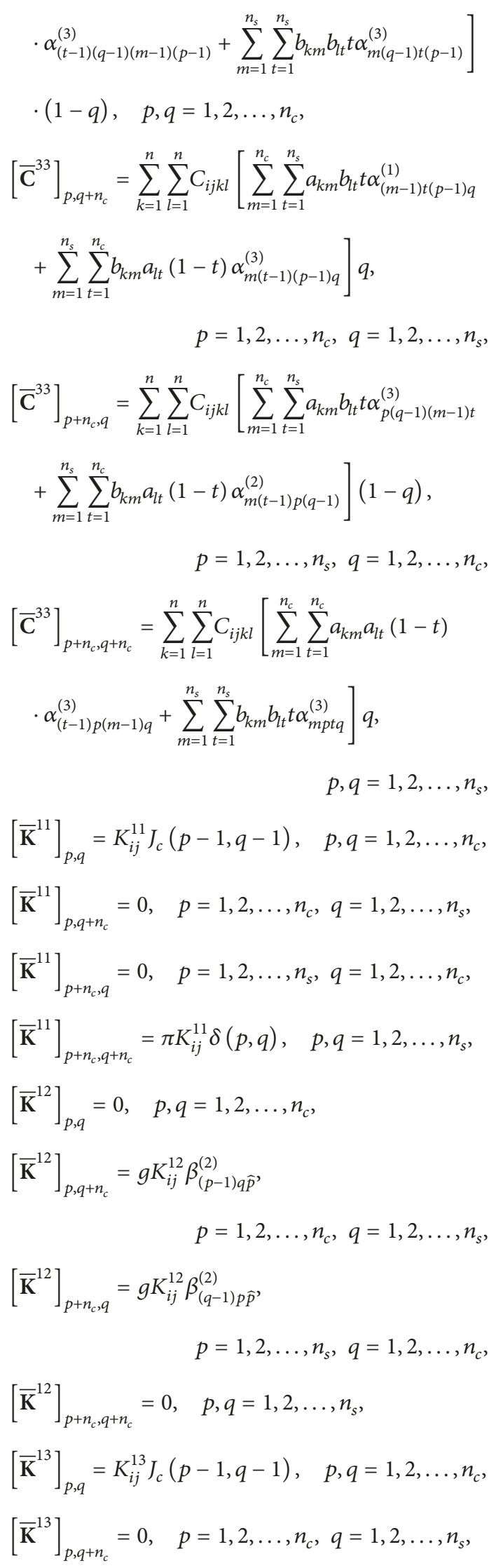


$\left[\overline{\mathbf{K}}^{13}\right]_{p+n_{c}, q}=0, \quad p=1,2, \ldots, n_{s}, q=1,2, \ldots, n_{c}$

$\left[\overline{\mathbf{K}}^{13}\right]_{p+n_{c}, q+n_{c}}=\pi K_{i j}^{13} \delta(p, q), \quad p, q=1,2, \ldots, n_{s}$

$\left[\overline{\mathbf{K}}^{21}\right]_{p, q}=g \sum_{k=1}^{n}\left(K_{i j k}+K_{i k j}\right)$

$\cdot \sum_{m=1}^{n_{c}}\left[a_{k m} \alpha_{(m-1)(p-1)(q-1) \hat{p}}^{(1)}\right], \quad p, q=1,2, \ldots, n_{c}$

$\left[\overline{\mathbf{K}}^{21}\right]_{p, q+n_{c}}=g \sum_{k=1}^{n}\left(K_{i j k}+K_{i k j}\right) \sum_{m=1}^{n_{s}}\left[b_{k m} \alpha_{m q(p-1) \hat{p}}^{(3)}\right]$,

$$
p=1,2, \ldots, n_{c}, q=1,2, \ldots, n_{s},
$$

$\left[\overline{\mathbf{K}}^{21}\right]_{p+n_{c}, q}=g \sum_{k=1}^{n}\left(K_{i j k}+K_{i k j}\right) \sum_{m=1}^{n_{s}}\left[b_{k m} \alpha_{m p(q-1) \hat{p}}^{(3)}\right]$,

$$
p=1,2, \ldots, n_{s}, q=1,2, \ldots, n_{c}
$$

$\left[\overline{\mathbf{K}}^{21}\right]_{p+n_{c}, q+n_{c}}=g \sum_{k=1}^{n}\left(K_{i j k}+K_{i k j}\right) \sum_{m=1}^{n_{c}}\left[a_{k m} \alpha_{p q(m-1) \hat{p}}^{(3)}\right]$,

$$
p, q=1,2, \ldots, n_{s}
$$

$\left[\overline{\mathbf{K}}^{22}\right]_{p, q}=g \sum_{k=1}^{n} K_{i j k} \sum_{m=1}^{n_{c}}\left[a_{k m} \alpha_{(m-1)(p-1)(q-1) \hat{p}}^{(1)}\right]$,

$$
p, q=1,2, \ldots, n_{c}
$$

$\left[\overline{\mathbf{K}}^{22}\right]_{p, q+n_{c}}=g \sum_{k=1}^{n} K_{i j k} \sum_{m=1}^{n_{s}}\left[b_{k m} \alpha_{m q(p-1) \hat{p}}^{(3)}\right]$,

$$
p=1,2, \ldots, n_{c}, q=1,2, \ldots, n_{s}
$$

$\left[\overline{\mathbf{K}}^{22}\right]_{p+n_{c}, q}=g \sum_{k=1}^{n} K_{i j k} \sum_{m=1}^{n_{s}}\left[b_{k m} \alpha_{m p(q-1) \hat{p}}^{(3)}\right]$,

$$
p=1,2, \ldots, n_{s}, q=1,2, \ldots, n_{c},
$$

$\left[\overline{\mathbf{K}}^{22}\right]_{p+n_{c}, q+n_{c}}=g \sum_{k=1}^{n} K_{i j k} \sum_{m=1}^{n_{c}}\left[a_{k m} \alpha_{p q(m-1) \hat{p}}^{(3)}\right]$,

$$
p, q=1,2, \ldots, n_{s}
$$

$\left[\overline{\mathbf{K}}^{31}\right]_{p, q}=\sum_{k=1}^{n} \sum_{l=1}^{n}\left(K_{i j k l}+K_{i l k j}+K_{i k j l}\right)$

$\cdot\left[\sum_{m=1}^{n_{c}} \sum_{t=1}^{n_{c}} a_{k m} a_{l t} \alpha_{(m-1)(t-1)(p-1)(q-1)}^{(1)}+\sum_{m=1}^{n_{s}} \sum_{t=1}^{n_{s}} b_{k m} b_{l t}\right.$

$\left.\cdot \alpha_{m t(p-1)(q-1)}^{(3)}\right], \quad p, q=1,2, \ldots, n_{c}$,

$\left[\overline{\mathbf{K}}^{31}\right]_{p, q+n_{c}}=2 \sum_{k=1}^{n} \sum_{l=1}^{n}\left(K_{i j k l}+K_{i l k j}+K_{i k j l}\right)$

$\cdot \sum_{m=1}^{n_{c}} \sum_{t=1}^{n_{s}} a_{k m} b_{l t} \alpha_{t q(m-1)(p-1)}^{(3)}$ $p=1,2, \ldots, n_{c}, q=1,2, \ldots, n_{s}$,

$\left[\overline{\mathbf{K}}^{31}\right]_{p+n_{c}, q}=2 \sum_{k=1}^{n} \sum_{l=1}^{n}\left(K_{i j k l}+K_{i l k j}+K_{i k j l}\right)$

$\sum_{m=1}^{n_{c}} \sum_{t=1}^{n_{s}} a_{k m} b_{l t} \alpha_{t p(m-1)(q-1)}^{(3)}$,

$$
p=1,2, \ldots, n_{s}, q=1,2, \ldots, n_{c},
$$

$\left[\overline{\mathbf{K}}^{31}\right]_{p+n_{c}, q+n_{c}}=\sum_{k=1}^{n} \sum_{l=1}^{n}\left(K_{i j k l}+K_{i l k j}+K_{i k j l}\right)$

$\cdot\left[\sum_{m=1}^{n_{c}} \sum_{t=1}^{n_{c}} a_{k m} a_{l t} \alpha_{p q(m-1)(t-1)}^{(3)}+\sum_{m=1}^{n_{s}} \sum_{t=1}^{n_{s}} b_{k m} b_{l t} \alpha_{m t p q}^{(2)}\right]$,

$p, q=1,2, \ldots, n_{s}$,

$\left[\overline{\mathbf{K}}^{32}\right]_{p, q}=\sum_{k=1}^{n} \sum_{l=1}^{n} K_{i j k l}$

$\cdot\left[\sum_{m=1}^{n_{c}} \sum_{t=1}^{n_{c}} a_{k m} a_{l t} \alpha_{(m-1)(t-1)(p-1)(q-1)}^{(1)}+\sum_{m=1}^{n_{s}} \sum_{t=1}^{n_{s}} b_{k m} b_{l t}\right.$

$\left.\cdot \alpha_{m t(p-1)(q-1)}^{(3)}\right], \quad p, q=1,2, \ldots, n_{c}$

$\left[\overline{\mathbf{K}}^{32}\right]_{p, q+n_{c}}=2 \sum_{k=1}^{n} \sum_{l=1}^{n} K_{i j k l} \sum_{m=1}^{n_{c}} \sum_{t=1}^{n_{s}} a_{k m} b_{l t} \alpha_{t q(m-1)(p-1)}^{(3)}$,

$p=1,2, \ldots, n_{c}, q=1,2, \ldots, n_{s}$,

$\left[\overline{\mathbf{K}}^{32}\right]_{p+n_{c}, q}=2 \sum_{k=1}^{n} \sum_{l=1}^{n} K_{i j k l} \sum_{m=1}^{n_{c}} \sum_{t=1}^{n_{s}} a_{k m} b_{l t} \alpha_{t p(m-1)(q-1)}^{(3)}$,

$p=1,2, \ldots, n_{s}, q=1,2, \ldots, n_{c}$,

$\left[\overline{\mathbf{K}}^{32}\right]_{p+n_{c}, q+n_{c}}=\sum_{k=1}^{n} \sum_{l=1}^{n} K_{i j k l}$

$\cdot\left[\sum_{m=1}^{n_{c}} \sum_{t=1}^{n_{c}} a_{k m} a_{l t} \alpha_{p q(m-1)(t-1)}^{(3)}+\sum_{m=1}^{n_{s}} \sum_{t=1}^{n_{s}} b_{k m} b_{l t} \alpha_{m t p q}^{(2)}\right]$,

$p, q=1,2, \ldots, n_{s}$,

$$
\overline{\mathbf{F}}=\left[\begin{array}{llll}
\bar{F}_{1} & \bar{F}_{2} & \cdots & \bar{F}_{n}
\end{array}\right]^{T},
$$

where $i=1,2, \ldots, n ; j=1,2, \ldots, n$; and

$$
\begin{aligned}
& \delta(p, q)= \begin{cases}1 & \text { if } p=q \\
0 & \text { if } p \neq q,\end{cases} \\
& \beta_{m p q}^{(1)}=\frac{1}{2}\left[J_{c}(m, p+q)+J_{c}(m, p-q)\right], \\
& \beta_{m p q}^{(2)}=\frac{1}{2}\left[J_{c}(m, p-q)-J_{c}(m, p+q)\right],
\end{aligned}
$$




$$
\begin{aligned}
& \alpha_{m t p q}^{(1)}=\frac{1}{4}\left[J_{c}(m+t, p+q)+J_{c}(m+t, p-q)\right. \\
& \left.+J_{c}(m-t, p+q)+J_{c}(m-t, p-q)\right], \\
& \alpha_{m t p q}^{(2)}=\frac{1}{4}\left[J_{c}(m-t, p-q)+J_{c}(m+t, p+q)\right. \\
& \left.-J_{c}(m+t, p-q)-J_{c}(m-t, p+q)\right], \\
& \alpha_{m t p q}^{(3)}=\frac{1}{4}\left[J_{c}(m-t, p+q)+J_{c}(m-t, p-q)\right. \\
& \left.-J_{c}(m+t, p+q)-J_{c}(m+t, p-q)\right],
\end{aligned}
$$

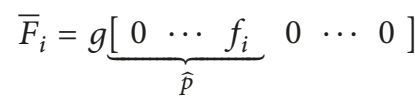

in which

$$
J_{c}=\int_{0}^{2 \pi} \cos m \tau \cos n \tau d \tau= \begin{cases}0 & m \neq n \\ 2 \pi & m=n=0 \\ \pi & m=n \neq 0 .\end{cases}
$$

\section{Conflicts of Interest}

The authors declare that they have no conflicts of interest.

\section{Acknowledgments}

Financial supports from the National Natural Science Foundation of China (Grant nos. 11572354 and 11442006), the Natural Science Foundation of Guangdong Province in China (Grant nos. 2016A030313328 and 2014A030313115), and the National Science Foundation (Grant no. CMMI-1335397) are gratefully acknowledged.

\section{References}

[1] F. Conrad and Ö. Morgül, "On the stabilization of a flexible beam with a tip mass," SIAM Journal on Control and Optimization, vol. 36, no. 6, pp. 1962-1986, 1998.

[2] G. V. Rao, "Linear dynamics of an elastic beam under moving loads," Journal of Vibration and Acoustics, vol. 122, no. 3, pp. 281289, 2000.

[3] M. A. Demetriou, "Natural observers for second order lumped and distributed parameter systems using parameter-dependent Lyapunov functions," in Proceedings of the 2001 American Control Conference, pp. 2503-2508, IEEE, Arlington, VA, USA, June 2001.

[4] S. D. Furta, "Linear vibrations of a rotating elastic beam with an attached point mass," Journal of Engineering Mathematics, vol. 46, no. 2, pp. 165-188, 2003.

[5] M. Ansari, E. Esmailzadeh, and N. Jalili, "Exact frequency analysis of a rotating cantilever beam with tip mass subjected to torsional-bending vibrations," Journal of Vibration and Acoustics, vol. 133, no. 4, Article ID 041003, 2011.

[6] H. Yang, J. Z. Hong, and Z. Y. Yu, "Dynamics modelling of a flexible hub-beam system with a tip mass," Journal of Sound and Vibration, vol. 266, no. 4, pp. 759-774, 2003.
[7] S. Z. Ismail, A. A. Al-Qaisia, and B. O. Al-Bedoor, "Dynamic model of a rotating flexible arm-flexible root mechanism driven by a shaft flexible in torsion," Shock and Vibration, vol. 13, no. 6, pp. 577-593, 2006.

[8] D. L. Gregory, F. Bitsie, and D. O. Smallwood, "Comparison of the response of a simple structure to single axis and multiple axis random vibration inputs," in Proceedings of the in Proceedings of the 79th Shock and Vibration Symposium, Orlando, Fla, USA, 2008.

[9] M. Sunar and B. O. Al-Bedoor, "Vibration measurement of rotating blades using a root embedded PZT sensor," Shock and Vibration, vol. 15, no. 5, pp. 517-541, 2008.

[10] A. Sinha, S. Bose, A. Nandi, and S. Neogy, "A precessing and nutating beam with a tip mass," Mechanics Research Communications, vol. 53, pp. 75-84, 2013.

[11] O. Patil and P. Gandhi, "On the dynamics and multiple equilibria of an inverted flexible pendulum with tip mass on a cart," Journal of Dynamic Systems, Measurement, and Control, vol. 136, no. 4, Article ID 041017, 2014.

[12] C. C. Li, X. Y. Meng, and Z. Z. Liu, "Dynamic modeling and simulation for the rigid flexible coupling system with a non-tip payload in non-inertial coordinate system," Journal of Vibration and Control, vol. 22, no. 4, pp. 1076-1094, 2016.

[13] Y. F. Yong, W. L. Yan, C. Hu, and W. J. Min, "Dynamic Modeling and Response of a Rotating Cantilever Beam with a Concentrated Mass," Shock and Vibration, vol. 2016, Article ID 8935247, 2016.

[14] E. H. K. Fung and Z. X. Shi, "Vibration frequencies of a constrained flexible arm carrying an end mass," Journal of Sound and Vibration, vol. 204, no. 2, pp. 259-269, 1997.

[15] J.-H. Park and J.-H. Kim, "Dynamic analysis of rotating curved beam with a tip mass," Journal of Sound and Vibration, vol. 228, no. 5, pp. 1017-1034, 1999.

[16] A. F. Lynch and D. Wang, "Flatness-based control of a flexible beam in a gravitational field," in Proceedings of the 2004 American Control Conference (AAC), pp. 5449-5454, IEEE, Boston, MA, USA, July 2004.

[17] G.-P. Cai, J.-Z. Hong, and S. X. Yang, "Dynamic analysis of a flexible hub-beam system with tip mass," Mechanics Research Communications, vol. 32, no. 2, pp. 173-190, 2005.

[18] S. A. Emam, "Dynamics of a flexible-hub geometrically nonlinear beam with a tip mass," Journal of Vibration and Control, vol. 16, no. 13, pp. 1989-2000, 2010.

[19] P. S. Gandhi, P. Borja, and R. Ortega, "Energy shaping control of an inverted flexible pendulum fixed to a cart," Control Engineering Practice, vol. 56, pp. 27-36, 2016.

[20] S. L. Lau and Y. K. Cheung, "Amplitude incremental variational principle for nonlinear vibration of elastic systems," Journal of Applied Mechanics, vol. 48, no. 4, pp. 959-964, 1981.

[21] S. L. Lau and Y. K. Cheung, "Incremental time-space finite strip method for non-linear structural vibrations," Earthquake Engineering \& Structural Dynamics, vol. 10, no. 2, pp. 239-253, 1982.

[22] G. Y. Xu and W. D. Zhu, "Nonlinear and time-varying dynamics of high-dimensional models of a translating beam with a stationary load subsystem," Journal of Vibration and Acoustics, vol. 132, no. 6, Article ID 061012, 2010.

[23] W. Lu, F. Ge, X. Wu, and Y. Hong, "Nonlinear dynamics of a submerged floating moored structure by incremental harmonic balance method with FFT,' Marine Structures, vol. 31, pp. 6381, 2013. 
[24] X. F. Wang and W. D. Zhu, "Dynamic Analysis of an Automotive Belt-Drive System with a Noncircular Sprocket by a Modified Incremental Harmonic Balance Method," Journal of Vibration and Acoustics, vol. 139, no. 1, Article ID 011009, 2017.

[25] W. D. Zhu, H. Ren, and C. Xiao, "A nonlinear model of a slack cable with bending stiffness and moving ends with application to elevator traveling and compensation cables," Journal of Applied Mechanics, vol. 78, no. 4, pp. 41017-41017-13, 2011.

[26] Y. K. Cheung, S. H. Chen, and S. L. Lau, "Application of the incremental harmonic balance method to cubic non-linearity systems," Journal of Sound and Vibration, vol. 140, no. 2, pp. 273286, 1990.

[27] E. H. K. Fung and D. T. W. Yau, "Vibration frequencies of a rotating flexible arm carrying a moving mass," Journal of Sound and Vibration, vol. 241, no. 5, pp. 857-878, 2001. 


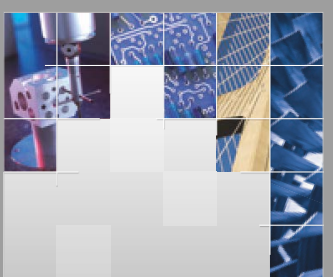

\section{Enfincering}
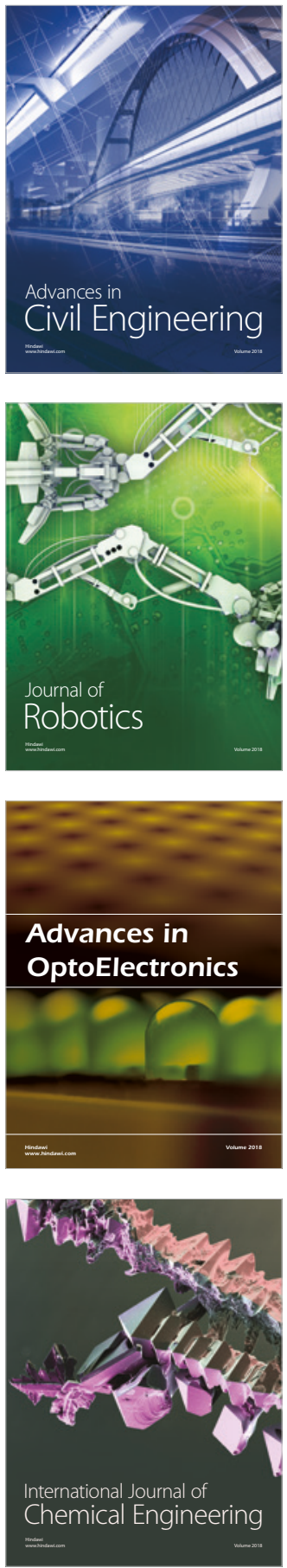

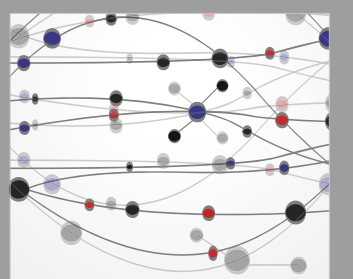

\section{Rotating \\ Machinery}

The Scientific World Journal

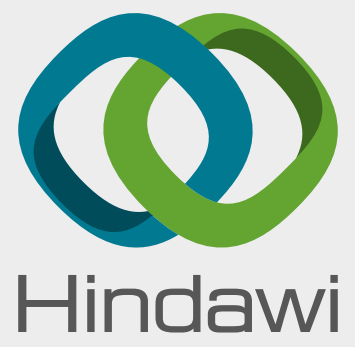

Submit your manuscripts at

www.hindawi.com
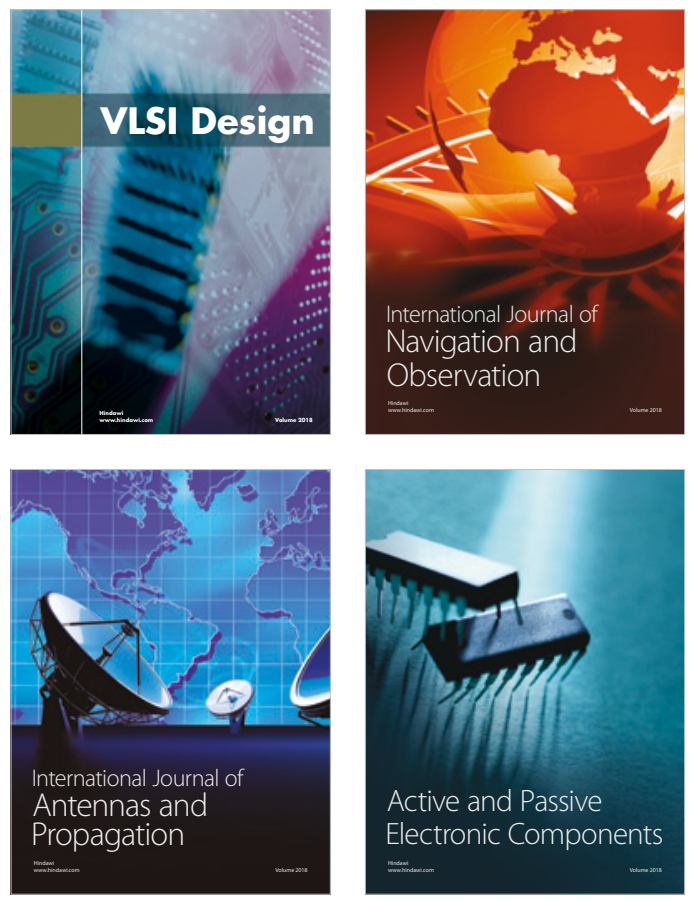


\section{Advances \\ Multimedia}
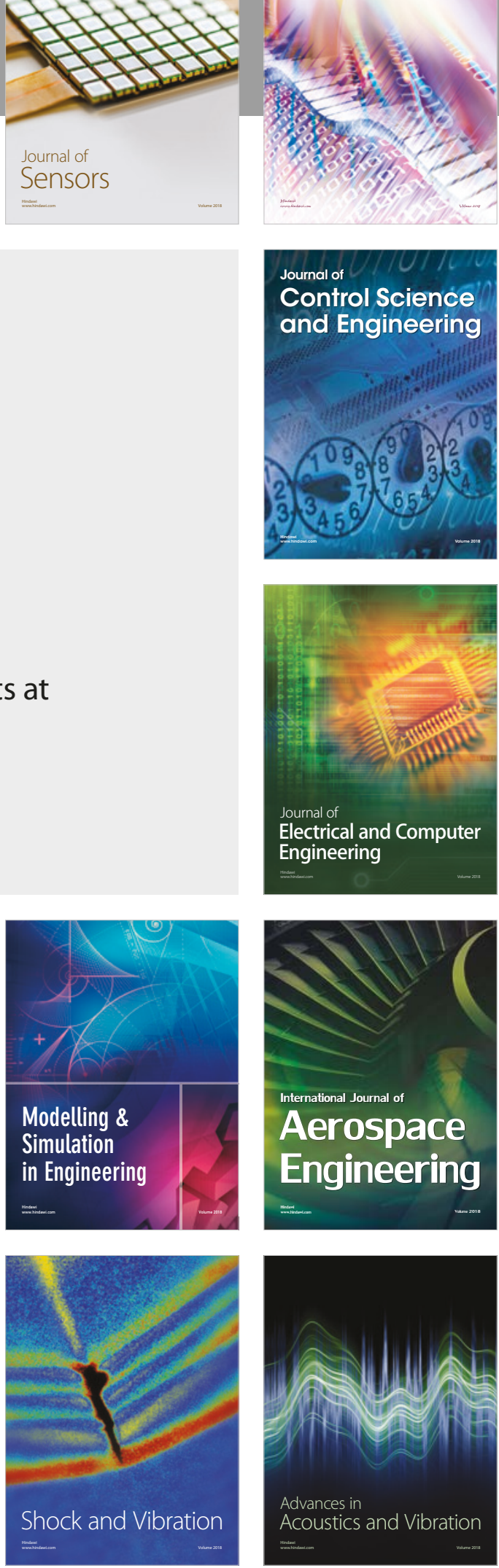